DOI 10.4171/JEMS/311

Michael Gekhtman · Michael Shapiro · Alek Vainshtein

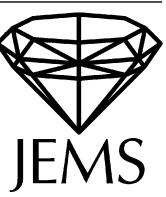

\title{
Poisson geometry of directed networks in an annulus
}

Received February 5, 2009 and in revised form November 30, 2010

\begin{abstract}
As a generalization of Postnikov's construction [P], we define a map from the space of edge weights of a directed network in an annulus into a space of loops in the Grassmannian. We then show that universal Poisson brackets introduced for the space of edge weights in [GSV3] induce a family of Poisson structures on rational matrix-valued functions and on the space of loops in the Grassmannian. In the former case, this family includes, for a particular kind of networks, the Poisson bracket associated with the trigonometric R-matrix.
\end{abstract}

\section{Introduction}

This is the second in a series of four papers initiated by [GSV3] and devoted to geometry behind directed networks on surfaces, with a particular emphasis on their Poisson properties.

In [GSV3], we concentrated on Postnikov's construction [P] that uses weighted directed planar graphs to parametrize cells in Grassmannians. We found that the space of edge weights of networks in a disk can be endowed with a natural family of Poisson brackets (which we called universal) that "respects" the operation of concatenation of diagrams. We have shown that, under Postnikov's parametrization, these Poisson brackets induce a two-parameter family of Poisson brackets on the Grassmannian. Every Poisson bracket in this family is compatible (in the sense of [GSV1, GSV2]) with the cluster algebra on the Grassmannian described in [GSV1, S] and, on the other hand, endows the Grassmannian with a structure of a Poisson homogeneous space with respect to the natural action of the general linear group equipped with an R-matrix Poisson-Lie structure.

As was announced in [GSV3], the current paper builds a parallel theory for directed weighted networks in an annulus (or, equivalently, on a cylinder). First, we have to modify the definition of the boundary measurement map, whose image now consists of rational

M. Gekhtman: Department of Mathematics, University of Notre Dame, Notre Dame, IN 46556, USA; e-mail: mgekhtma@nd.edu

M. Shapiro: Department of Mathematics, Michigan State University, East Lansing, MI 48823, USA; e-mail:mshapiro@math.msu.edu

A. Vainshtein: Department of Mathematics AND Department of Computer Science, University of Haifa, Haifa, Mount Carmel 31905, Israel; e-mail: alek@cs.haifa.ac.il

Mathematics Subject Classification (2010): 53D17, 14M15 
matrix-valued functions of an auxiliary parameter $\lambda$ associated with the notion of a $c u t$ (see Section 1). We then show that the analogue of Postnikov's construction leads to a map into the space of loops in the Grassmannian. Universal Poisson brackets for networks in an annulus are defined in exactly the same way as in the case of a disk. We show that they induce a two-parameter family of Poisson brackets on boundary measurements. In particular, when all sources are located on one of the boundary circles, and all sinks on the other, one of the generators of this family coincides with the Sklyanin R-matrix bracket associated with the trigonometric solution of the classical Yang-Baxter equation in $\operatorname{sl}(n)$. Moreover, we prove that the two-parameter family of Poisson brackets can be further pushed forward to the space of loops in the Grassmannian. In proving the latter, we departed from the approach of [GSV3] where a similar result was obtained via a more or less straightforward calculation. Such an approach would have been too cumbersome in our current setting. Instead, we found a way to utilize so-called face weights and their behavior under path-reversal maps.

The third paper in this series [GSV4] focuses on particular graphs in an annulus that can be used to introduce a cluster algebra structure on the coordinate ring of the space of normalized rational functions in one variable. This space is birationally equivalent, via the Moser map [M], to any minimal irreducible coadjoint orbit of the group of upper triangular matrices associated with a Coxeter element of the permutation group. In this case, the Poisson bracket compatible with the cluster algebra structure coincides with the quadratic Poisson bracket studied in [FG1, FG2] in the context of Toda flows on minimal orbits. We show that cluster transformations serve as Bäcklund-Darboux transformations between different minimal Toda flows. The fourth paper [GSV5] solves, in the case of graphs in an annulus, the inverse problem of restoring the weights from the image of the generalized Postnikov map. In the case of arbitrary planar graphs in a disk, this problem was completely solved by Postnikov [P] who proved that for a fixed minimal graph, the space of weights modulo gauge action is birational to its image. In contrast to this case, already for simplest graphs in an annulus, the corresponding map can only be shown to be finite, that is, the number of collections of weights that produce the same boundary measurement for a fixed minimal graph is finite.

The original application of directed weighted planar networks was in the study of total positivity, both in $G L_{n}$ [KM, B, BFZ, FZ, Fa] and in Grassmannians [P]. We do not investigate this aspect for networks in an annulus. It has been studied in a recent preprint [LP].

The paper is organized as follows.

In Section 2, we introduce the notion of a perfect network in an annulus and associate with every such network a matrix of boundary measurements. Each boundary measurement is shown to be a rational function in edge weights and in an auxiliary parameter $\lambda$ (see Corollary 2.3). Moreover, we define the space of face and trail weights, a generalization of the space of face weights studied in [GSV3] for the case of networks in a disk, and provide its cohomological interpretation (see Section 2.3).

In Section 3, we characterize all universal Poisson brackets on the space of edge weights of a given network that respect the natural operation of concatenation of networks (see Proposition 3.1). Furthermore, we establish that the family of universal brack- 
ets induces a linear two-parameter family of Poisson brackets on the space of face and trail weights (see Theorem 3.2), and hence on boundary measurement matrices (see Theorem 3.3). This family depends on a relative location of sources and sinks, but not on the network itself. We provide an explicit description of this family in Propositions 3.4 and 3.6. An important tool in the proof of Theorem 3.3 is the realization theorem (see Theorem 3.8) that states that any rational matrix function can be realized as the boundary measurement matrix of a network with a given set of sources and sinks. Finally, if the sources and the sinks are separated, that is, all sources belong to one of the bounding circles of the annulus, and all sinks to the other bounding circle, one of the generators of the two-parameter family can be identified with the R-matrix Sklyanin bracket corresponding to the trigonometric R-matrix (see Theorem 3.13).

In Section 4 , the boundary measurement map defined by a network with $k$ sources, $n-k$ sinks and $n_{1} \leq n$ boundary vertices on the outer boundary circle is extended to the Grassmannian boundary measurement map into the space $L G_{k}(n)$ of Grassmannian loops. The Poisson family on boundary measurement matrices allows us to equip $L G_{k}(n)$ with a two-parameter family of Poisson brackets $\mathcal{P}_{\alpha, \beta}^{n_{1}}$ in such a way that for any choice of a universal Poisson bracket on edge weights there is a unique member of $\mathcal{P}_{\alpha, \beta}^{n_{1}}$ that makes the Grassmannian boundary measurement map Poisson (see Theorem 4.7). This latter family depends only on the number of sources and sinks and on the distribution of the boundary vertices between the bounding circles of the annulus. The main tool in the proof of Theorem 4.7 is the path reversal operation on networks and its properties (see Theorem 4.1).

\section{Perfect planar networks and boundary measurements}

\subsection{Networks, cuts, paths and weights}

Let $G=(V, E)$ be a directed planar graph drawn inside an annulus with the vertex set $V$ and the edge set $E$. Exactly $n$ of its vertices are located on the boundary circles of the annulus and are called boundary vertices; $n_{1} \geq 0$ of them lie on the outer circle, and $n_{2}=n-n_{1} \geq 0$ on the inner circle. The graph is considered up to an isotopy relative to the boundary (with fixed boundary vertices).

Each boundary vertex is marked as a source or a sink. A source is a vertex with exactly one outcoming edge and no incoming edges. Sinks are defined in the same way, with the direction of the single edge reversed. All the internal vertices of $G$ have degree 3 and are of two types: either they have exactly one incoming edge, or exactly one outcoming edge. The vertices of the first type are called (and shown in figures) white, those of the second type, black.

A cut $\rho$ is an oriented non-selfintersecting curve starting at a base point on the inner circle and ending at a base point on the outer circle considered up to an isotopy relative to the boundary (with fixed endpoints). We assume that the base points of the cut are distinct from the boundary vertices of $G$. For an arbitrary oriented curve $\gamma$ with endpoints not lying on the cut $\rho$ we denote by ind $(\gamma)$ the algebraic intersection number of $\gamma$ and $\rho$. Recall that each transversal intersection point of $\gamma$ and $\rho$ contributes 1 to this number if 
the oriented tangents to $\gamma$ and $\rho$ at this point form a positively oriented basis, and -1 otherwise. Non-transversal intersection points are treated in a similar way.

Let $x_{1}, \ldots, x_{d}$ be independent variables. A perfect planar network in an annulus $N=$ $(G, \rho, w)$ is obtained from a graph $G$ equipped with a cut $\rho$ as above by assigning a weight $w_{e} \in \mathbb{Z}\left(x_{1}, \ldots, x_{d}\right)$ to each edge $e \in E$. Below we occasionally write "network" instead of "perfect planar network in an annulus". Each network defines a rational map $w: \mathbb{R}^{d} \rightarrow \mathbb{R}^{|E|}$; the space of edge weights $\mathcal{E}_{N}$ is defined as the intersection of the image of $w$ with $(\mathbb{R} \backslash 0)^{|E|}$. In other words, a point in $\mathcal{E}_{N}$ is a graph $G$ as above with edges weighted by non-zero real numbers obtained by specializing the variables $x_{1}, \ldots, x_{d}$ in the expressions for $w_{e}$.

A path $P$ in $N$ is an alternating sequence $\left(v_{1}, e_{1}, v_{2}, \ldots, e_{r}, v_{r+1}\right)$ of vertices and edges such that $e_{i}=\left(v_{i}, v_{i+1}\right)$ for any $i \in[1, r]$. Sometimes we omit the names of the vertices and write $P=\left(e_{1}, \ldots, e_{r}\right)$. A path is called a cycle if $v_{r+1}=v_{1}$, and a simple cycle if additionally $v_{i} \neq v_{j}$ for any other pair $i \neq j$.

To define the weight of a path we need the following construction. Consider a closed oriented polygonal plane curve $C$. Let $e^{\prime}$ and $e^{\prime \prime}$ be two consecutive oriented segments of $C$, and let $v$ be their common vertex. We assume for simplicity that for any such pair $\left(e^{\prime}, e^{\prime \prime}\right)$, the cone spanned by $e^{\prime}$ and $e^{\prime \prime}$ is not a line; in other words, if $e^{\prime}$ and $e^{\prime \prime}$ are collinear, then they have the same direction. Observe that since $C$ is not necessarily simple, there might be other edges of $C$ incident to $v$. Let $l$ be an arbitrary oriented line. Define $c_{l}\left(e^{\prime}, e^{\prime \prime}\right) \in \mathbb{Z} / 2 \mathbb{Z}$ in the following way: $c_{l}\left(e^{\prime}, e^{\prime \prime}\right)=1$ if the directing vector of $l$ belongs to the interior of the cone spanned by $e^{\prime}$ and $e^{\prime \prime}$, and $c_{l}\left(e^{\prime}, e^{\prime \prime}\right)=0$ otherwise. Define $c_{l}(C)$ as the sum of $c_{l}\left(e^{\prime}, e^{\prime \prime}\right)$ over all pairs of consecutive segments in $C$. It follows immediately from Theorem 1 in [GrSh] that $c_{l}(C)$ does not depend on $l$, provided $l$ is not collinear to any of the segments in $C$. The common value of $c_{l}(C)$ for different choices of $l$ is denoted by $c(C)$ and called the concordance number of $C$. In fact, $c(C)$ equals mod 2 the rotation number of $C$; the definition of the latter is similar, but more complicated.

In what follows we assume without loss of generality that $N$ is drawn in such a way that all its edges and the cut are smooth curves. Moreover, any simple path in $N$ is a piecewise-smooth curve with no cusps, at any boundary vertex of $N$ the edge and the circle intersect transversally, and the same holds for the cut at both of its base points. Given a path $P$ between a source $b^{\prime}$ and a sink $b^{\prime \prime}$, we define a closed piecewise-smooth curve $C_{P}$ in the following way: if both $b^{\prime}$ and $b^{\prime \prime}$ belong to the same circle, $C_{P}$ is obtained by adding to $P$ the path between $b^{\prime \prime}$ and $b^{\prime}$ that goes counterclockwise along the boundary of the corresponding circle. Otherwise, if $b^{\prime}$ and $b^{\prime \prime}$ belong to distinct circles, $C_{P}$ is obtained by adding to $P$ the path that starts at $b^{\prime \prime}$, goes counterclockwise along the corresponding circle to the base point of the cut, follows the cut to the other base point and then goes counterclockwise along the other circle up to $b^{\prime}$. Clearly, the resulting curve $C_{P}$ does not have cusps, so its concordance number $c\left(C_{P}\right)$ can be defined in a straightforward manner via polygonal approximation.

Finally the weight of $P$ is defined as

$$
w_{P}=w_{P}(\lambda)=(-1)^{c\left(C_{P}\right)-1} \lambda^{\operatorname{ind}(P)} \prod_{e \in P} w_{e},
$$


where $\lambda$ is an auxiliary independent variable. Occasionally, it will be convenient to assume that the internal vertices of $G$ do not lie on the cut and to rewrite the above formula as

$$
w_{P}=(-1)^{c\left(C_{P}\right)-1} \prod_{e \in P} \bar{w}_{e},
$$

where $\bar{w}_{e}=w_{e} \lambda^{\text {ind(e) }}$ are modified edge weights. Observe that the weight of a path is a relative isotopy invariant, while modified edge weights are not. The weight of an arbitrary cycle in $N$ is defined in the same way via the concordance number of the cycle.

If edges $e_{i}$ and $e_{j}$ in $P$ coincide and $i<j$, the path $P$ can be decomposed into the path $P^{\prime}=\left(e_{1}, \ldots, e_{i-1}, e_{i}=e_{j}, e_{j+1}, \ldots, e_{r}\right)$ and the cycle $C^{0}=\left(e_{i}, e_{i+1}, \ldots, e_{j-1}\right)$. Clearly, $c\left(C_{P}\right)=c\left(C_{P^{\prime}}\right)+c\left(C^{0}\right)$, and hence

$$
w_{P}=-w_{P^{\prime}} w_{C^{0}} .
$$

An example of a perfect planar network in an annulus is shown in Fig. 2.1 (left). It has two sources, $b$ on the outer circle and $b^{\prime \prime}$ on the inner circle, and one sink $b^{\prime}$ on the inner circle. Each edge $e_{i}$ is labeled by its weight. The cut is shown by the dashed line. The same network is shown in Fig. 2.1 (right); it differs from the original picture by an isotopic deformation of the cut.
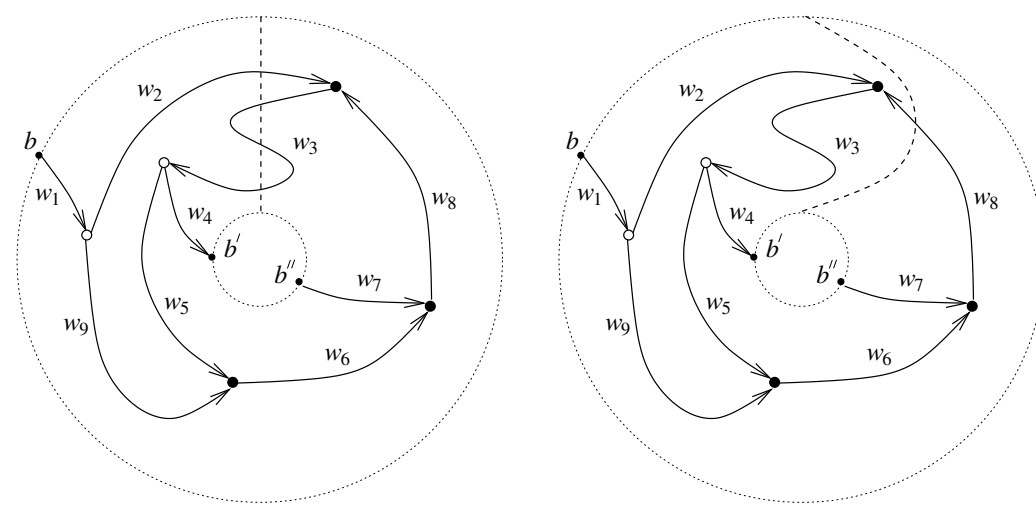

Fig. 2.1. A perfect planar network in an annulus.

Consider the path $P_{1}=\left(e_{1}, e_{2}, e_{3}, e_{4}\right)$ from $b$ to $b^{\prime}$. Its algebraic intersection number with the cut equals 0 (in the left picture, two intersection points contribute 1 each, and two other intersection points contribute -1 each; in the right picture there are no intersection points). The concordance number of the corresponding closed curve $C_{P_{1}}$ equals 1 . Therefore, (2.1) gives $w_{P_{1}}=w_{1} w_{2} w_{3} w_{4}$. On the other hand, the modified weights for the left picture are given by $\bar{w}_{1}=w_{1}, \bar{w}_{2}=\lambda w_{2}, \bar{w}_{3}=\lambda^{-1} w_{3}, \bar{w}_{4}=w_{4}$, and hence computation via (2.2) gives $w_{P_{1}}=\bar{w}_{1} \bar{w}_{2} \bar{w}_{3} \bar{w}_{4}=w_{1} w_{2} w_{3} w_{4}$. Finally, the modified weights for the relevant edges in the right picture coincide with the original weights, and we again get the same result.

Consider the path $P_{2}=\left(e_{7}, e_{8}, e_{3}, e_{4}\right)$ from $b^{\prime \prime}$ to $b^{\prime}$. Its algebraic intersection number with the cut equals -1 , and the concordance number of the corresponding closed curve 
$C_{P_{2}}$ equals 1. Therefore, $w_{P_{2}}=\lambda^{-1} w_{3} w_{4} w_{7} w_{8}$. The same result can be obtained by using modified weights.

Finally, consider the path $P_{3}=\left(e_{1}, e_{2}, e_{3}, e_{5}, e_{6}, e_{8}, e_{3}, e_{4}\right)$ from $b$ to $b^{\prime}$. Clearly, $w_{P_{3}}=-\lambda^{-1} w_{1} w_{2} w_{3}^{2} w_{4} w_{5} w_{6} w_{8}$. The path $P_{3}$ can be decomposed into the path $P_{1}$ as above and a cycle $C^{0}=\left(e_{3}, e_{5}, e_{6}, e_{8}\right)$ with the weights $w_{P_{1}}=w_{1} w_{2} w_{3} w_{4}$ and $w_{C^{0}}=\lambda^{-1} w_{3} w_{5} w_{6} w_{8}$, hence relation (2.3) yields the same expression for $w_{P_{3}}$ as before.

Let us see how moving a base point of the cut affects the weights of paths. Let $N=(G, \rho, w)$ and $N^{\prime}=\left(G, \rho^{\prime}, w\right)$ be two networks with the same graph and the same weights, and assume that the cuts $\rho$ and $\rho^{\prime}$ are not isotopic. More exactly, let us start moving a base point of the cut in the counterclockwise direction. Assume that $b$ is the first boundary vertex in the counterclockwise direction from the base point of $\rho$ that is being moved. Clearly, nothing is changed while the base point and $b$ do not interchange. Let $\rho^{\prime}$ be the cut obtained after the interchange, and assume that no other interchanges occurred. Then the relation between the weight $w_{P}$ of a path $P$ in $N$ and its weight $w_{P}^{\prime}$ in $N^{\prime}$ is given by the following proposition.

Proposition 2.1. For $N$ and $N^{\prime}$ as above,

$$
w_{P}^{\prime}(\lambda)=\left((-1)^{\alpha(P)} \lambda\right)^{\beta(b, P)} w_{P},
$$

where $\alpha(P)$ equals 0 if the endpoints of $P$ lie on the same circle and 1 otherwise, and

$$
\beta(b, P)= \begin{cases}1 & \text { if } b \text { is the sink of } P, \\ -1 & \text { if } b \text { is the source of } P \\ 0 & \text { otherwise. }\end{cases}
$$

Proof. The proof is straightforward.

For example, consider the networks $N$ and $N^{\prime}$ shown in Fig. 2.2. The base point of the cut lying on the inner circle interchanges positions with the sink $b^{\prime}$. The path $P_{1}$ from the previous example goes from the outer circle to the inner circle, so $\alpha\left(P_{1}\right)=1$;

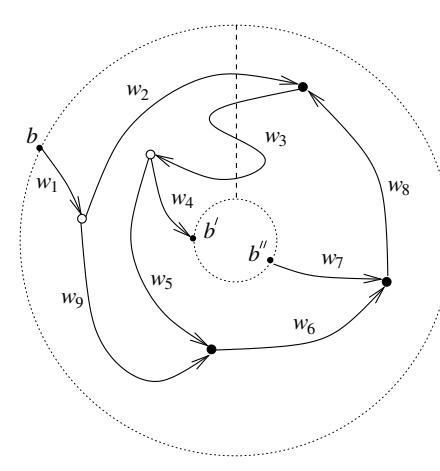

$N$

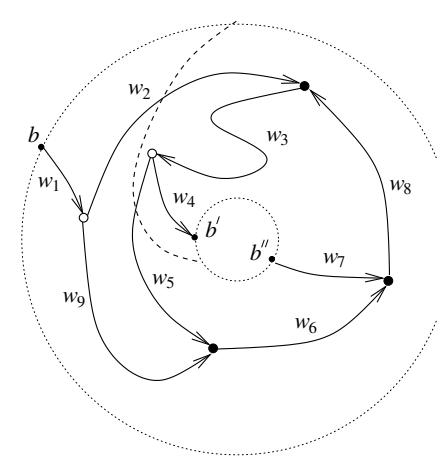

$N^{\prime}$

Fig. 2.2. Moving the base point of the cut. 
moreover, $b^{\prime}$ is the sink of $P_{1}$, so $\beta\left(b^{\prime}, P_{1}\right)=1$. Therefore, by Proposition $2.1, w_{P_{1}}^{\prime}=$ $-\lambda w_{P_{1}}=-\lambda w_{1} w_{2} w_{3} w_{4}$, which coincides with the value obtained via (2.2). The path $P_{2}$ from the previous example starts and ends at the inner circle, so $\alpha\left(P_{2}\right)=0$. Moreover, $\beta\left(b^{\prime}, P_{2}\right)=1$, and hence, by Proposition 2.1, $w_{P_{2}}^{\prime}=\lambda w_{P_{2}}=w_{1} w_{2} w_{3} w_{4}$; once again, this coincides with the value obtained via (2.2).

\subsection{Boundary measurements}

Given a perfect planar network in an annulus as above, we label its boundary vertices $b_{1}, \ldots, b_{n}$ in the following way. The boundary vertices lying on the outer circle are labeled $b_{1}, \ldots, b_{n_{1}}$ in the counterclockwise order starting from the first vertex that occurs after the base point of the cut. The boundary vertices lying on the inner circle are labeled $b_{n_{1}+1}, \ldots, b_{n}$ in the clockwise order starting from the first vertex that occurs after the base point of the cut. For example, for the network $N$ in Fig. 2.2, the boundary vertices are labeled $b_{1}=b, b_{2}=b^{\prime \prime}, b_{3}=b^{\prime}$, while for the network $N^{\prime}$, one has $b_{1}=b, b_{2}=b^{\prime}$, $b_{3}=b^{\prime \prime}$.

The number of sources lying on the outer circle is denoted by $k_{1}$, and the corresponding set of indices, by $I_{1} \subset\left[1, n_{1}\right]$; the set of the remaining $m_{1}=n_{1}-k_{1}$ indices is denoted by $J_{1}$. Similarly, the number of sources lying on the inner circle is denoted by $k_{2}$, and the corresponding set of indices, by $I_{2} \subset\left[n_{1}+1, n\right]$; the set of the remaining $m_{2}=n_{2}-k_{2}$ indices is denoted by $J_{2}$. Finally, we denote $I=I_{1} \cup I_{2}$ and $J=J_{1} \cup J_{2}$; the cardinalities of $I$ and $J$ are denoted $k=k_{1}+k_{2}$ and $m=m_{1}+m_{2}$.

Given a source $b_{i}, i \in I$, and a sink $b_{j}, j \in J$, we define the boundary measurement $M(i, j)$ as the sum of the weights of all paths starting at $b_{i}$ and ending at $b_{j}$. Assume first that the weights of the paths are calculated via (2.2). The boundary measurement thus defined is a formal infinite series in variables $\bar{w}_{e}, e \in E$. The following proposition holds true in $\mathbb{Z}\left[\left[\bar{w}_{e}, e \in E\right]\right]$.

Proposition 2.2. Let $N$ be a perfect planar network in an annulus. Then each boundary measurement in $N$ is a rational function in the modified weights $\bar{w}_{e}, e \in E$.

Proof. The proof by induction on the number of internal vertices literally follows the proof of the similar statement in [GSV3]. The only changes are that modified weights are used instead of original weights and that the counterclockwise cyclic order $\prec$ is replaced by the cyclic order mod $n$ induced by the labeling.

Taking into account the definition of the modified weights, we immediately get the following corollary.

Corollary 2.3. Let $N$ be a perfect planar network in an annulus. Then each boundary measurement in $N$ is a rational function in the parameter $\lambda$ and in the weights $w_{e}, e \in E$.

For example, the boundary measurement $M(1,2)$ in the network $N^{\prime}$ shown in Fig. 2.2 equals

$$
\frac{w_{1} w_{3} w_{4}\left(w_{6} w_{8} w_{9}-\lambda w_{2}\right)}{1+\lambda^{-1} w_{3} w_{5} w_{6} w_{8}}
$$


Boundary measurements can be organized into a $k \times m$ boundary measurement matrix $M_{N}$ exactly as in the case of planar networks in the disk. Let $I=\left\{i_{1}<\cdots<i_{k}\right\}$ and $J=$ $\left\{j_{1}<\cdots<j_{m}\right\}$. Then $M_{N}=\left(M_{p q}\right), p \in[1, k], q \in[1, m]$, where $M_{p q}=M\left(i_{p}, j_{q}\right)$. Let $\mathrm{Rat}_{k, m}$ stand for the space of real rational $k \times m$ matrix functions in one variable. Then each network $N$ defines a map $\mathcal{E}_{N} \rightarrow$ Rat $_{k, m}$ given by $M_{N}$ and called the boundary measurement map corresponding to $N$.

The boundary measurement matrix has a block structure

$$
M_{N}=\left(\begin{array}{ll}
M_{1} & M_{2} \\
M_{3} & M_{4}
\end{array}\right)
$$

where $M_{1}$ is $k_{1} \times m_{1}$ and $M_{4}$ is $k_{2} \times m_{2}$. Moving a base point of the cut changes the weights of paths as described in Proposition 2.1, which affects $M_{N}$ in the following way. Define

$$
\Lambda_{+}=\left(\begin{array}{cccc}
0 & 1 & \ldots & 0 \\
\vdots & \vdots & \ddots & \vdots \\
0 & 0 & \ldots & 1 \\
\lambda^{-1} & 0 & \ldots & 0
\end{array}\right), \quad \Lambda_{-}=\left(\begin{array}{cccc}
0 & 1 & \ldots & 0 \\
\vdots & \vdots & \ddots & \vdots \\
0 & 0 & \ldots & 1 \\
-\lambda^{-1} & 0 & \ldots & 0
\end{array}\right)
$$

Then interchanging the base point of the cut with $b_{1}$ implies the transformation

$$
\left(\begin{array}{ll}
M_{1} & M_{2} \\
M_{3} & M_{4}
\end{array}\right) \mapsto\left(\begin{array}{cc}
\Lambda_{+} M_{1} & \Lambda_{-} M_{2} \\
M_{3} & M_{4}
\end{array}\right)
$$

if $b_{1}$ is a source, and

$$
\left(\begin{array}{ll}
M_{1} & M_{2} \\
M_{3} & M_{4}
\end{array}\right) \mapsto\left(\begin{array}{ll}
M_{1} \Lambda_{+}^{-1} & M_{2} \\
M_{3} \Lambda_{-}^{-1} & M_{4}
\end{array}\right)
$$

if $b_{1}$ is a sink, while interchanging the base point of the cut with $b_{n}$ yields

$$
\left(\begin{array}{cc}
M_{1} & M_{2} \\
M_{3} & M_{4}
\end{array}\right) \mapsto\left(\begin{array}{cc}
M_{1} & M_{2} \\
\Lambda_{+}^{T} M_{3} & \Lambda_{-}^{T} M_{4}
\end{array}\right)
$$

if $b_{n}$ is a source, and

$$
\left(\begin{array}{ll}
M_{1} & M_{2} \\
M_{3} & M_{4}
\end{array}\right) \mapsto\left(\begin{array}{ll}
M_{1} & M_{2}\left(\Lambda_{+}^{-1}\right)^{T} \\
M_{3} & M_{4}\left(\Lambda_{-}^{-1}\right)^{T}
\end{array}\right)
$$

if $b_{1}$ is a sink. Note that $\Lambda_{+}$and $\Lambda_{-}$are $k_{1} \times k_{1}$ in the first case, $m_{1} \times m_{1}$ in the second case, $k_{2} \times k_{2}$ in the third case and $m_{2} \times m_{2}$ in the fourth case.

\subsection{The space of face and trail weights}

Let $N=(G, \rho, w)$ be a perfect network. Consider the $\mathbb{Z}$-module $\mathbb{Z}^{E}$ generated by the edges of $G$. Clearly, points of $\mathcal{E}_{N}$ can be identified with elements of $\operatorname{Hom}\left(\mathbb{Z}^{E}, \mathbb{R}^{*}\right)$ via $w\left(\sum n_{i} e_{i}\right)=\prod w_{e_{i}}^{n_{i}}$, where $\mathbb{R}^{*}$ is the abelian multiplicative group $\mathbb{R} \backslash 0$. Further, consider 
the $\mathbb{Z}$-module $\mathbb{Z}^{V}$ generated by the vertices of $G$ and its $\mathbb{Z}$-submodule $\mathbb{Z}^{V_{0}}$ generated by the internal vertices. An arbitrary element $\varphi \in \operatorname{Hom}\left(\mathbb{Z}^{V}, \mathbb{R}^{*}\right)$ acts on $\mathcal{E}_{N}$ as follows: if $e=(u, v)$ then

$$
w_{e} \mapsto w_{e} \frac{\varphi(v)}{\varphi(u)} .
$$

Therefore, the weight of a path between the boundary vertices $b_{i}$ and $b_{j}$ is multiplied by $\varphi\left(b_{j}\right) / \varphi\left(b_{i}\right)$. It follows that the gauge group $\mathfrak{G}$, which preserves the weights of all paths between boundary vertices, consists of all $\varphi \in \operatorname{Hom}\left(\mathbb{Z}^{V}, \mathbb{R}^{*}\right)$ such that $\varphi(b)=1$ for any boundary vertex $b$, and can be identified with $\operatorname{Hom}\left(\mathbb{Z}^{V_{0}}, \mathbb{R}^{*}\right)$. Thus, the boundary measurement map $\mathcal{E}_{N} \rightarrow$ Rat $_{k, m}$ factors through the quotient space $\mathcal{F}_{N}=\mathcal{E}_{N} / \mathfrak{G}$ as follows: $M_{N}=M_{N}^{\mathcal{F}} \circ y$, where $y: \mathcal{E}_{N} \rightarrow \mathcal{F}_{N}$ is the projection and $M_{N}^{\mathcal{F}}$ is a map $\mathcal{F}_{N} \rightarrow$ Rat $_{k, m}$. The space $\mathcal{F}_{N}$ is called the space of face and trail weights for the following reasons.

First, by considering the cochain complex

$$
0 \rightarrow \mathfrak{G} \rightarrow \mathcal{E}_{N} \rightarrow 0
$$

with the coboundary operator $\delta: \mathfrak{G} \rightarrow \mathcal{E}_{N}$ defined by $\delta(\varphi)(e)=\varphi(v) / \varphi(u)$ for $e=$ $(u, v)$, we can identify $\mathcal{F}_{N}$ with the first relative cohomology $H^{1}\left(G, \partial G ; \mathbb{R}^{*}\right)$ of the complex, where $\partial G$ is the set of all boundary vertices of $G$.

Second, consider a slightly more general situation, when the annulus is replaced by an arbitrary Riemann surface $\Sigma$ of genus zero with the boundary $\partial \Sigma$, and $G$ is embedded into $\Sigma$ in such a way that all vertices of degree 1 belong to $\partial \Sigma$ (boundary vertices). Then the exact sequence of relative cohomology with coefficients in $\mathbb{R}^{*}$ gives

$$
\begin{aligned}
0 \rightarrow H^{0}(G \cup \partial \Sigma, \partial \Sigma) & \rightarrow H^{0}(G \cup \partial \Sigma) \rightarrow H^{0}(\partial \Sigma) \\
& \rightarrow H^{1}(G \cup \partial \Sigma, \partial \Sigma) \rightarrow H^{1}(G \cup \partial \Sigma) \rightarrow H^{1}(\partial \Sigma) \rightarrow 0 .
\end{aligned}
$$

Evidently, $H^{1}(G, \partial G) \simeq H^{1}(G \cup \partial \Sigma, \partial \Sigma)$. Next, $H^{0}(G \cup \partial \Sigma, \partial \Sigma)=0$, since each connected component of $G$ is connected to at least one connected component of $\partial \Sigma$, and hence

$$
H^{1}(G, \partial G) \simeq H^{1}(G \cup \partial \Sigma) / H^{1}(\partial \Sigma) \oplus H^{0}(\partial \Sigma) / H^{0}(G \cup \partial \Sigma)=\mathcal{F}_{N}^{f} \oplus \mathcal{F}_{N}^{t} .
$$

The space $\mathcal{F}_{N}^{f}$ can be described as follows. The graph $G$ divides $\Sigma$ into a finite number of connected components called faces. The boundary of each face consists of edges of $G$ and, possibly, of several arcs of $\partial \Sigma$. A face is called bounded if its boundary contains only edges of $G$, and unbounded otherwise.

Given a face $f$, we define its face weight $y_{f}$ as the function on $\mathcal{E}_{N}$ that assigns to the edge weights $w_{e}, e \in E$, the value

$$
y_{f}=\prod_{e \in \partial f} w_{e}^{\gamma_{e}},
$$

where $\gamma_{e}=1$ if the direction of $e$ is compatible with the counterclockwise orientation of the boundary $\partial f$, and $\gamma_{e}=-1$ otherwise. It follows immediately from the definition that 
face weights are invariant under the gauge group action, and hence are functions on $\mathcal{F}_{N}^{f}$, and moreover form a basis in the space of such functions.

Consider now the space $\mathcal{F}_{N}^{t}$. If $\Sigma$ is a disk, then $\mathcal{F}_{N}^{t}=0$, and hence $\mathcal{F}_{N}$ and $\mathcal{F}_{N}^{f}$ coincide. This case was studied in [GSV3], and the space $\mathcal{F}_{N}$ was called there the space of face weights. If $\Sigma$ is an annulus, two cases are possible. Indeed, $\operatorname{dim} H^{0}(\partial \Sigma)=2$. The dimension of $H^{0}(G \cup \partial \Sigma)$ is either 2 or 1, depending on the existence of a trail connecting the components of $\partial \Sigma$. Here a trail is a sequence $\left(v_{1}, \ldots, v_{k+1}\right)$ of vertices such that either $\left(v_{i}, v_{i+1}\right)$ or $\left(v_{i+1}, v_{i}\right)$ is an edge in $G$ for all $i \in[1, k]$ and the endpoints $v_{1}$ and $v_{k+1}$ are boundary vertices of $G$. Given a trail $t$, the trail weight $y_{t}$ is defined as

$$
y_{t}=\prod_{i=1}^{k} w\left(v_{i}, v_{i+1}\right),
$$

where

$$
w\left(v_{i}, v_{i+1}\right)= \begin{cases}w_{e} & \text { if } e=\left(v_{i}, v_{i+1}\right) \in E, \\ w_{e}^{-1} & \text { if } e=\left(v_{i+1}, v_{i}\right) \in E .\end{cases}
$$

Clearly, the trail weights are invariant under the action of the gauge group.

If $G$ does not contain a trail connecting the inner and the outer circles, then $\operatorname{dim} H^{0}(G \cup \partial \Sigma)=2$, and hence $\mathcal{F}_{N}^{t}=0$. Otherwise, $\operatorname{dim} H^{0}(G \cup \partial \Sigma)=1$, and hence $\operatorname{dim} \mathcal{F}_{N}^{t}=1$. The functions on $\mathcal{F}_{N}^{t}$ are generated by the weight of any connecting trail.

\section{Poisson properties of the boundary measurement map}

\subsection{Poisson structures on the spaces $\mathcal{E}_{N}$ and $\mathcal{F}_{N}$}

The construction of a Poisson structure on the space $\mathcal{E}_{N}$ for perfect planar networks in an annulus is a straightforward extension of the corresponding construction for the case of the disk studied in [GSV3]. Let $G$ be a directed planar graph in an annulus as described in Section 2.1. A pair $(v, e)$ is called a flag if $v$ is an endpoint of $e$. To each internal vertex $v$ of $G$ we assign a 3 -dimensional space $(\mathbb{R} \backslash 0)_{v}^{3}$ with coordinates $x_{v}^{1}, x_{v}^{2}, x_{v}^{3}$. We equip each $(\mathbb{R} \backslash 0)_{v}^{3}$ with a Poisson bracket $\{\cdot, \cdot\}_{v}$. It is convenient to assume that the flags involving $v$ are labeled by the coordinates, as shown in Figure 3.1.
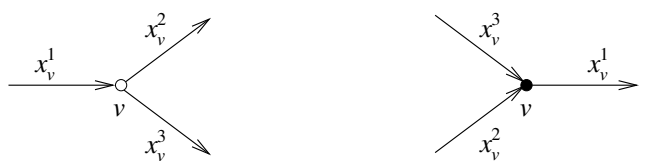

Fig. 3.1. Edge labeling for $\mathbb{R}_{v}^{3}$.

Moreover, to each boundary vertex $b_{j}$ of $G$ we assign a 1 -dimensional space $(\mathbb{R} \backslash 0)_{j}$ with the coordinate $x_{j}^{1}$ (in accordance with the above convention, this coordinate labels the unique flag involving $b_{j}$ ). Define $\mathcal{R}$ to be the direct sum of all the above spaces; thus, 
the dimension of $\mathcal{R}$ equals twice the number of edges in $G$. Note that $\mathcal{R}$ is equipped with a Poisson bracket $\{\cdot, \cdot\}_{\mathcal{R}}$, which is defined as the direct sum of the brackets $\{\cdot, \cdot\}_{v}$; that is, $\{x, y\}_{\mathcal{R}}=0$ whenever $x$ and $y$ are not defined on the same $(\mathbb{R} \backslash 0)_{v}^{3}$. We say that the bracket $\{\cdot, \cdot\}_{\mathcal{R}}$ is universal if each of $\{\cdot, \cdot\}_{v}$ depends only on the color of the vertex $v$.

Define the weights $w_{e}$ by

$$
w_{e}=x_{v}^{i} x_{u}^{j}
$$

provided the flag $(v, e)$ is labeled by $x_{v}^{i}$ and the flag $(u, e)$ is labeled by $x_{u}^{j}$. In other words, the weight of an edge is defined as the product of the weights of the two flags involving this edge. Therefore, in this case the space of edge weights $\mathcal{E}_{N}$ coincides with the entire $(\mathbb{R} \backslash 0)^{|E|}$, and the weights define a weight map $w:(\mathbb{R} \backslash 0)^{d} \rightarrow(\mathbb{R} \backslash 0)^{|E|}$. We require the pushforward of $\{\cdot, \cdot\}_{\mathcal{R}}$ to $(\mathbb{R} \backslash 0)^{|E|}$ by the weight map to be a well defined Poisson bracket; this can be regarded as an analog of the Poisson-Lie property for groups.

Proposition 3.1. Universal Poisson brackets $\{\cdot, \cdot\}_{\mathcal{R}}$ such that the weight map $w$ is Poisson form a 6-parameter family defined by the relations

$$
\left\{x_{v}^{i}, x_{v}^{j}\right\}_{v}=\alpha_{i j} x_{v}^{i} x_{v}^{j}, \quad i, j \in[1,3], i \neq j,
$$

at each white vertex $v$ and

$$
\left\{x_{v}^{i}, x_{v}^{j}\right\}_{v}=\beta_{i j} x_{v}^{i} x_{v}^{j}, \quad i, j \in[1,3], i \neq j,
$$

at each black vertex $v$.

Proof. Indeed, let $v$ be a white vertex, and let $e=(v, u)$ and $\bar{e}=(v, \bar{u})$ be the two outcoming edges. By definition, there exist $i, j, k, l \in[1,3], i \neq j$, such that $w_{e}=x_{v}^{i} x_{u}^{k}$, $w_{\bar{e}}=x_{v}^{j} x_{\bar{u}}^{l}$. Therefore,

$$
\left\{w_{e}, w_{\bar{e}}\right\}_{N}=\left\{x_{v}^{i} x_{u}^{k}, x_{v}^{j} x_{\bar{u}}^{l}\right\}_{\mathcal{R}}=x_{u}^{k} x_{\bar{u}}^{l}\left\{x_{v}^{i}, x_{v}^{j}\right\}_{v},
$$

where $\{\cdot, \cdot\}_{N}$ stands for the pushforward of $\{\cdot, \cdot\}_{\mathcal{R}}$. Recall that the Poisson bracket in $(\mathbb{R} \backslash 0)_{v}^{3}$ depends only on $x_{v}^{1}, x_{v}^{2}$ and $x_{v}^{3}$. Hence the only possibility for the right hand side of the above relation to be a function of $w_{e}$ and $w_{\bar{e}}$ occurs when $\left\{x_{v}^{i}, x_{v}^{j}\right\}_{v}=\alpha_{i j} x_{v}^{i} x_{v}^{j}$, as required.

Black vertices are treated in the same way.

The 6-parameter family of universal Poisson brackets described in Proposition 3.1 induces a 6-parameter family of Poisson brackets $\{\cdot, \cdot\}_{N}$ on $\mathcal{E}_{N}$. Our next goal is to study the pushforward of this family to $\mathcal{F}_{N}$ by the map $y$.

Theorem 3.2. The 6-parameter family $\{\cdot, \cdot\}_{N}$ induces a 2-parameter family of Poisson brackets $\{\cdot, \cdot\}_{\mathcal{F}_{N}}$ on $\mathcal{F}_{N}$ with parameters $\alpha$ and $\beta$ given by

$$
\alpha=\alpha_{23}+\alpha_{13}-\alpha_{12}, \quad \beta=\beta_{23}+\beta_{13}-\beta_{12}
$$


Proof. In what follows it will be convenient to assume that boundary vertices are colored gray. Let $e=(u, v)$ be a directed edge. We say that the flag $(u, e)$ is positive, and the flag $(v, e)$ is negative. The color of a flag is defined as the color of the vertex participating in the flag.

Consider first a bracket of two face weights. Let $f$ and $f^{\prime}$ be two faces of $N$. We say that a flag $(v, e)$ is common to $f$ and $f^{\prime}$ if both $v$ and $e$ belong to $\partial f \cap \partial f^{\prime}$. Clearly, the bracket $\left\{y_{f}, y_{f^{\prime}}\right\}_{\mathcal{F}_{N}}$ can be calculated as the sum of the contributions of all flags common to $f$ and $f^{\prime}$.

Assume that $(v, e)$ is a positive white flag common to $f$ and $f^{\prime}$ (see Fig. 3.2). Then

$$
y_{f}=\frac{x_{v}^{3}}{x_{v}^{2}} \bar{y}_{f} \quad \text { and } \quad y_{f^{\prime}}=x_{v}^{1} x_{v}^{2} \bar{y}_{f^{\prime}},
$$

where $x_{v}^{i}$ are the weights of flags involving $v$ and $\left\{x_{v}^{i}, \bar{y}_{f}\right\}_{R}=\left\{x_{v}^{i}, \bar{y}_{f^{\prime}}\right\}_{R}=0$. Therefore, by (3.2), the contribution of $(v, e)$ equals $\left(\alpha_{12}-\alpha_{13}-\alpha_{23}\right) y_{f} y_{f^{\prime}}$, which by (3.4) equals $-\alpha y_{f} y_{f^{\prime}}$.

Assume now that $(v, e)$ is a negative white flag common to $f$ and $f^{\prime}$ (see Fig. 3.2). In this case

$$
y_{f}=\frac{1}{x_{v}^{1} x_{v}^{3}} \bar{y}_{f} \quad \text { and } \quad y_{f^{\prime}}=x_{v}^{1} x_{v}^{2} \bar{y}_{f^{\prime}},
$$

so the contribution of $(v, e)$ equals $\left(\alpha_{13}+\alpha_{23}-\alpha_{12}\right) y_{f} y_{f^{\prime}}=\alpha y_{f} y_{f^{\prime}}$.

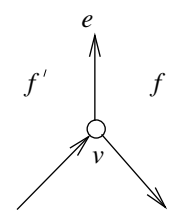

a)

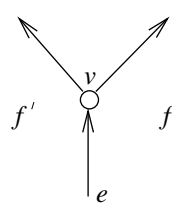

b)

Fig. 3.2. Contribution of a white common flag: a) positive flag; b) negative flag.

In a similar way one proves that the contribution of a positive black flag common to $f$ and $f^{\prime}$ equals $-\beta y_{f} y_{f^{\prime}}$, and the contribution of a negative black flag common to $f$ and $f^{\prime}$ equals $\beta y_{f} y_{f^{\prime}}$. Finally, the contributions of positive and negative gray flags are clearly equal to zero. Therefore, the brackets $\left\{y_{f}, y_{f^{\prime}}\right\}_{\mathcal{F}_{N}}$ form a 2-parameter family with parameters $\alpha$ and $\beta$ defined by (3.4).

The case of a bracket $\left\{y_{f}, y_{t}\right\}_{\mathcal{F}_{N}}$ is treated in a similar way.

\subsection{Induced Poisson structures on $\mathrm{Rat}_{k, m}$}

Fix an arbitrary pair of partitions $I_{1} \cup J_{1}=\left[1, n_{1}\right], I_{1} \cap J_{1}=\emptyset, I_{2} \cup J_{2}=\left[n_{1}+1, n\right]$, $I_{2} \cap J_{2}=\emptyset$, and denote $k=\left|I_{1}\right|+\left|I_{2}\right|, m=n-k=\left|J_{1}\right|+\left|J_{2}\right|$. Let $\operatorname{Net}_{I_{1}, J_{1}, I_{2}, J_{2}}$ stand for the set of all perfect planar networks in an annulus with the sources $b_{i}, i \in I_{1}$ and sinks $b_{j}, j \in J_{1}$, on the outer circle, sources $b_{i}, i \in I_{2}$ and sinks $b_{j}, j \in J_{2}$, on the inner 
circle, and edge weights $w_{e}$ defined by (3.1). We assume that the space of edge weights $\mathcal{E}_{N}=(\mathbb{R} \backslash 0)^{|E|}$ is equipped with the Poisson bracket $\{\cdot, \cdot\}_{N}$ obtained as the pushforward of the 6-parameter family $\{\cdot, \cdot\}_{R}$ described in Proposition 3.1.

Theorem 3.3. There exists a 2-parameter family of Poisson brackets $\{\cdot, \cdot\}_{I_{1}, J_{1}, I_{2}, J_{2}}$ on $\mathrm{Rat}_{k, m}$ with the following property: for any choice of parameters $\alpha_{i j}$, $\beta_{i j}$ in (3.2), (3.3) this family contains a unique Poisson bracket on $\mathrm{Rat}_{k, m}$ such that for any network $N \in$ Net $_{I_{1}, J_{1}, I_{2}, J_{2}}$ the map $M_{N}:(\mathbb{R} \backslash 0)^{|E|} \rightarrow$ Rat $_{k, m}$ is Poisson.

Proof. First of all, we use the factorization $M_{N}=M_{N}^{\mathcal{F}} \circ y$ to decrease the number of parameters. By Theorem 3.2, it suffices to consider the 2-parameter family

$$
\left\{\bar{x}_{v}^{2}, \bar{x}_{v}^{3}\right\}_{v}=\alpha \bar{x}_{v}^{2} \bar{x}_{v}^{3}, \quad\left\{\bar{x}_{v}^{1}, \bar{x}_{v}^{2}\right\}_{v}=\left\{\bar{x}_{v}^{1}, \bar{x}_{v}^{3}\right\}_{v}=0
$$

and

$$
\left\{\bar{x}_{v}^{2}, \bar{x}_{v}^{3}\right\}_{v}=\beta \bar{x}_{v}^{2} \bar{x}_{v}^{3}, \quad\left\{\bar{x}_{v}^{1}, \bar{x}_{v}^{2}\right\}_{v}=\left\{\bar{x}_{v}^{1}, \bar{x}_{v}^{3}\right\}_{v}=0
$$

with $\alpha$ and $\beta$ defined by (3.4), instead of the 6-parameter family (3.2), (3.3)

The rest of the proof consists of two major steps. First, we compute the induced Poisson bracket on the image of the boundary measurement map. More exactly, we show that the bracket $\{\cdot, \cdot\}_{N}$ of any pair of pullbacks of coordinate functions on the image can be expressed in terms of pullbacks of other coordinate functions, and that for fixed $I_{1}, J_{1}, I_{2}, J_{2}$ these expressions do not depend on the network $N \in \operatorname{Net}_{I_{1}, J_{1}, I_{2}, J_{2}}$. Second, we prove that any rational matrix function belongs to the image of the boundary measurement map (for a sufficiently large $N \in \operatorname{Net}_{I_{1}, J_{1}, I_{2}, J_{2}}$, and therefore $\{\cdot, \cdot\}_{N}$ induces $\{\cdot, \cdot\}_{I_{1}, J_{1}, I_{2}, J_{2}}$ on $\mathrm{Rat}_{k, m}$. This approach allows us to circumvent technical difficulties one encounters when attempting to check the Jacobi identity in the image in a straightforward way.

To compute the induced Poisson bracket on the image of the boundary measurement map, we consider the coordinate functions val $t: f \mapsto f(t)$ that assign to any $f \in$ Rat $_{1,1}$ its value at the point $t$. Given a pair of two matrix entries, it suffices to calculate the bracket between an arbitrary pair of functions val ${ }_{t}$ and val ${ }_{s}$ defined on two copies of Rat $t_{1,1}$ representing these entries. Since the pullback of $\mathrm{val}_{t}$ is the corresponding component of $M_{N}(t)$, we have to deal with expressions of the form $\left\{M_{p q}(t), M_{\bar{p} \bar{q}}(s)\right\}_{N}$.

To avoid overcomplicated formulas we consider separately two particular representatives of the family (3.5), (3.6): 1) $\alpha=-\beta=1$, and 2) $\alpha=\beta=1$. Any member of the family can be represented as a linear combination of the above two.

Denote by $\{\cdot, \cdot\}_{N}^{1}$ the member of the 2-parameter family (3.5), (3.6) corresponding to the case $\alpha=-\beta=1$. Moreover, define $\sigma_{=}\left(i, j, i^{\prime}, j^{\prime}\right)=\operatorname{sign}\left(i^{\prime}-i\right)-\operatorname{sign}\left(j^{\prime}-j\right)$; clearly, $\sigma_{=}\left(i, j, i^{\prime}, j^{\prime}\right)$ is closely related to $s_{=}\left(i, j, i^{\prime}, j^{\prime}\right)$ defined and studied in [GSV3]. The bracket induced by $\{\cdot, \cdot\}_{N}^{1}$ on the image of the boundary measurement map is completely described by the following statement.

Proposition 3.4. (i) Let $i_{p}, i_{\bar{p}} \in\left[1, n_{1}\right]$ and $1 \leq \max \left\{i_{p}, i_{\bar{p}}\right\}<j_{\bar{q}}<j_{q} \leq n$. Then

$$
\left\{M_{p q}(t), M_{\bar{p} \bar{q}}(s)\right\}_{N}^{1}=\sigma_{=}\left(i_{p}, j_{q}, i_{\bar{p}}, j_{\bar{q}}\right) M_{p \bar{q}}(s) M_{\bar{p} q}(t)-\frac{2}{t-s} \Phi_{p q}^{\bar{p} \bar{q}}(t, s),
$$


where

$$
\Phi_{p q}^{\bar{p} \bar{q}}(t, s)= \begin{cases}\left(M_{p \bar{q}}(t)-M_{p \bar{q}}(s)\right)\left(s M_{\bar{p} q}(t)-t M_{\bar{p} q}(s)\right), & j_{\bar{q}}<j_{q} \leq n_{1} \\ s M_{\bar{p} q}(t)\left(M_{p \bar{q}}(t)-M_{p \bar{q}}(s)\right), & j_{\bar{q}} \leq n_{1}<j_{q} \\ s\left(M_{p \bar{q}}(t) M_{\bar{p} q}(s)-M_{p \bar{q}}(s) M_{\bar{p} q}(t)\right), & n_{1}<j_{\bar{q}}<j_{q}\end{cases}
$$

(ii) Let $j_{\bar{q}}, j_{q} \in\left[n_{1}+1, n\right]$ and $1 \leq i_{p}<i_{\bar{p}}<\min \left\{j_{\bar{q}}, j_{q}\right\} \leq n$. Then

$$
\left\{M_{p q}(t), M_{\bar{p} \bar{q}}(s)\right\}_{N}^{1}=\sigma_{=}\left(i_{p}, j_{q}, i_{\bar{p}}, j_{\bar{q}}\right) M_{p \bar{q}}(t) M_{\bar{p} q}(s)-\frac{2}{t-s} \Psi_{p q}^{\bar{p}} \bar{q}(t, s),
$$

where

$$
\Psi_{p q}^{\bar{p} \bar{q}}(t, s)= \begin{cases}t\left(M_{p \bar{q}}(t) M_{\bar{p} q}(s)-M_{p \bar{q}}(s) M_{\bar{p} q}(t)\right), & i_{p}<i_{\bar{p}} \leq n_{1}, \\ -t M_{p \bar{q}}(t)\left(M_{\bar{p} q}(t)-M_{\bar{p} q}(s)\right), & i_{p} \leq n_{1}<i_{\bar{p}}, \\ -\left(t M_{p \bar{q}}(t)-s M_{p \bar{q}}(s)\right)\left(M_{\bar{p} q}(t)-M_{\bar{p} q}(s)\right), & n_{1}<i_{p}<i_{\bar{p}}\end{cases}
$$

(iii) Let $1 \leq i_{p}=i_{\bar{p}}<j_{q}=j_{\bar{q}} \leq n$. Then

$$
\begin{aligned}
& \left\{M_{p q}(t), M_{p q}(s)\right\}_{N}^{1} \\
& \quad= \begin{cases}-\frac{2}{t-s}\left(M_{p q}(t)-M_{p q}(s)\right)\left(s M_{p q}(t)-t M_{p q}(s)\right), & i_{p}<j_{q} \leq n_{1}, \\
0, & i_{p} \leq n_{1}<j_{q} .\end{cases}
\end{aligned}
$$

(iv) Let $1 \leq i_{p}<\min \left\{i_{\bar{p}}, j_{q}, j_{\bar{q}}\right\}$. Then

$$
\begin{aligned}
& \left\{M_{p q}(t), M_{\bar{p} \bar{q}}(s)\right\}_{N}^{1} \\
& \quad= \begin{cases}\frac{2 t}{t-s}\left(M_{p \bar{q}}(t)-M_{p \bar{q}}(s)\right)\left(M_{\bar{p} q}(t)-M_{\bar{p} q}(s)\right), & j_{\bar{q}}<i_{\bar{p}}<j_{q} \leq n_{1}, \\
-\frac{2 t}{t-s}\left(M_{p \bar{q}}(t) M_{\bar{p} q}(t)-M_{p \bar{q}}(s) M_{\bar{p} q}(s)\right), & j_{q} \leq n_{1}<i_{\bar{p}}<j_{\bar{q}}, \\
0, & j_{\bar{q}} \leq n_{1}<i_{\bar{p}}<j_{q} .\end{cases}
\end{aligned}
$$

Proof. Let us first make sure that relations (3.7)-(3.10) indeed allow one to compute $\left\{M_{p q}(t), M_{\bar{p} \bar{q}}(s)\right\}_{N}^{1}$ for any $p, \bar{p} \in[1, k]$ and $q, \bar{q} \in[1, m]$. This is done by employing the following three techniques:

- moving a base point of the cut;

- reversing the direction of the cut;

- reversing the orientation of boundary circles.

The first of the above techniques has been described in detail in Section 2.1. For example, let $1 \leq j_{q}<i_{p}<i_{\bar{p}}<j_{\bar{q}} \leq n_{1}$. This case is not covered explicitly by (3.7)-(3.10). Consider the network $N^{\prime}$ obtained from $N$ by moving the base point of the cut on the outer circle counterclockwise and interchanging it with $j_{q}$. In this new network one has $1 \leq i_{p^{\prime}}<i_{\bar{p}^{\prime}}<j_{\bar{q}^{\prime}}<j_{q}^{\prime}=n_{1}$, so the conditions of Proposition 3.4(i) are satisfied and (3.7) yields

$$
\begin{aligned}
\left\{M_{p^{\prime} q^{\prime}}(t), M_{\bar{p}^{\prime} \bar{q}^{\prime}}(s)\right\}_{N^{\prime}}^{1}= & 2 M_{p^{\prime} \bar{q}^{\prime}}(s) M_{\bar{p}^{\prime} q^{\prime}}(t) \\
& -\frac{2}{t-s}\left(M_{p^{\prime} \bar{q}^{\prime}}(t)-M_{p^{\prime} \bar{q}^{\prime}}(s)\right)\left(s M_{\bar{p}^{\prime} q^{\prime}}(t)-t M_{\bar{p}^{\prime} q^{\prime}}(s)\right) .
\end{aligned}
$$


By Lemma 2.1,

$M_{p^{\prime} q^{\prime}}(t)=t M_{p q}(t), \quad M_{\bar{p}^{\prime} \bar{q}^{\prime}}(t)=M_{\bar{p} \bar{q}}(t), \quad M_{p^{\prime} \bar{q}^{\prime}}(t)=M_{p \bar{q}}(t), \quad M_{\bar{p}^{\prime} q^{\prime}}(t)=t M_{\bar{p}^{\prime} q^{\prime}}(t)$.

Finally, $\{\cdot, \cdot\}_{N}=\{\cdot, \cdot\}_{N^{\prime}}$ for any pair of edge weights, so we get

$\left\{M_{p q}(t), M_{\bar{p} \bar{q}}(s)\right\}_{N}^{1}=2 M_{p \bar{q}}(s) M_{\bar{p} q}(t)-\frac{2 s}{t-s}\left(M_{p \bar{q}}(t)-M_{p \bar{q}}(s)\right)\left(M_{\bar{p} q}(t)-M_{\bar{p} q}(s)\right)$.

Reversing the direction of the cut transforms the initial network $N$ to a new network $N^{\prime}$; the graph $G$ remains the same, while the labeling of its boundary vertices is changed. Namely, the $n_{1}^{\prime}=n_{2}$ boundary vertices lying on the inner circle are labeled $b_{1}, \ldots, b_{n_{1}^{\prime}}$ in the clockwise order starting from the first vertex that occurs after the base point of the cut. The boundary vertices lying on the outer circle are labeled $b_{n_{1}^{\prime}+1}, \ldots, b_{n}$ in the counterclockwise order starting from the first vertex that occurs after the base point of the cut. The transformation $N \mapsto N^{\prime}$ is better visualized if the network is drawn on a cylinder, instead of an annulus. The boundary circles of a cylinder are identical, and reversing the direction of the cut simply interchanges them. Clearly, the boundary measurements in $N$ and $N^{\prime}$ are related by $M_{r^{\prime} s^{\prime}}(t)=M_{r s}(1 / t)$ for any $i_{r} \in I, j_{s} \in J$. Moreover, $\{\cdot, \cdot\}_{N}=\{\cdot, \cdot\}_{N^{\prime}}$ for any pair of edge weights. Therefore, an expression for $\left\{M_{p q}(t), M_{\bar{p} \bar{q}}(s)\right\}_{N}^{1}$ via $M_{p \bar{q}}(t), M_{p \bar{q}}(s), M_{\bar{p} q}(t), M_{\bar{p} q}(s)$ is transformed to the expression for $\left\{M_{p^{\prime} q^{\prime}}(t), M_{\bar{p}^{\prime} \bar{q}^{\prime}}(s)\right\}_{N^{\prime}}^{1}$ via $M_{p^{\prime} \bar{q}^{\prime}}(t), M_{p^{\prime} \bar{q}^{\prime}}(s), M_{\bar{p}^{\prime} q^{\prime}}(t)$, $M_{\bar{p}^{\prime} q^{\prime}}(s)$ by the substitution $t \mapsto 1 / t$ and $s \mapsto 1 / s$ in the coefficients. For example, let $1 \leq i_{\bar{p}}<j_{q}<j_{\bar{q}} \leq n_{1}<i_{p} \leq n$. This case is not covered explicitly by (3.7)(3.10). Consider the network $N^{\prime}$ obtained from $N$ by reversing the direction of the cut. In this new network one has $1 \leq i_{p^{\prime}} \leq n_{1}^{\prime}<i_{\bar{p}^{\prime}}<j_{q}<j_{\bar{q}^{\prime}} \leq n$, so the conditions of Proposition 3.4(ii) are satisfied and (3.8) yields

$$
\left\{M_{p^{\prime} q^{\prime}}(t), M_{\bar{p}^{\prime} \bar{q}^{\prime}}(s)\right\}_{N^{\prime}}^{1}=-2 M_{p^{\prime} \bar{q}^{\prime}}(t) M_{\bar{p}^{\prime} q^{\prime}}(s)+\frac{2 t}{t-s} M_{p^{\prime} \bar{q}^{\prime}}(t)\left(M_{\bar{p}^{\prime} q^{\prime}}(t)-M_{\bar{p}^{\prime} q^{\prime}}(s)\right) .
$$

Applying the above described rule one gets

$$
\begin{aligned}
\left\{M_{p q}(t), M_{\bar{p} \bar{q}}(s)\right\}_{N}^{1} & =-2 M_{p \bar{q}}(t) M_{\bar{p} q}(s)+\frac{2 t^{-1}}{t^{-1}-s^{-1}} M_{p \bar{q}}(t)\left(M_{\bar{p} q}(t)-M_{\bar{p} q}(s)\right) \\
& =-2 M_{p \bar{q}}(t) M_{\bar{p} q}(s)-\frac{2 s}{t-s} M_{p \bar{q}}(t)\left(M_{\bar{p} q}(t)-M_{\bar{p} q}(s)\right) .
\end{aligned}
$$

Finally, reversing the orientation of boundary circles also retains the graph $G$ and changes the labeling of its boundary vertices. Namely, the $n_{1}$ boundary vertices of $N^{\prime}$ lying on the outer circle are labeled $b_{1}, \ldots, b_{n_{1}}$ in the clockwise order starting from the first vertex that occurs after the base point of the cut. The boundary vertices lying on the inner circle are labeled $b_{n_{1}+1}, \ldots, b_{n}$ in the counterclockwise order starting from the first vertex that occurs after the base point of the cut. The transformation $N \mapsto N^{\prime}$ may be visualized as a mirror reflection. Clearly, the boundary measurements in $N$ and $N^{\prime}$ are related by $M_{r^{\prime} s^{\prime}}(t)=M_{r s}(1 / t)$ for any $i_{r} \in I, j_{s} \in J$. Moreover, $\{\cdot, \cdot\}_{N}=$ $-\{\cdot, \cdot\}_{N^{\prime}}$ for any pair of edge weights. Therefore, the transformation of the expressions 
for the brackets differs from the one for the case of cut reversal by a factor of -1 . For example, let $1 \leq j_{q}<i_{p}<i_{\bar{p}}<j_{\bar{q}} \leq n_{1}$. This case is not covered explicitly by (3.7)(3.10). Consider the network $N^{\prime}$ obtained from $N$ by reversing the orientation of boundary circles. In this new network one has $1 \leq i_{p^{\prime}}<j_{\bar{q}^{\prime}}<i_{\bar{p}^{\prime}}<j_{q} \leq n_{1}$, so the conditions of Proposition 3.4(iv) are satisfied and (3.10) yields

$$
\left\{M_{p^{\prime} q^{\prime}}(t), M_{\bar{p}^{\prime} \bar{q}^{\prime}}(s)\right\}_{N^{\prime}}^{1}=\frac{2 t}{t-s}\left(M_{p^{\prime} \bar{q}^{\prime}}(t)-M_{p^{\prime} \bar{q}^{\prime}}(s)\right)\left(M_{\bar{p}^{\prime} q^{\prime}}(t)-M_{\bar{p}^{\prime} q^{\prime}}(s)\right) .
$$

Applying the above described rule one gets

$$
\begin{aligned}
\left\{M_{p q}(t), M_{\bar{p} \bar{q}}(s)\right\}_{N}^{1} & =-\frac{2 t^{-1}}{t^{-1}-s^{-1}}\left(M_{p \bar{q}}(t)-M_{p \bar{q}}(s)\right)\left(M_{\bar{p} q}(t)-M_{\bar{p} q}(s)\right) \\
& =\frac{2 s}{t-s}\left(M_{p \bar{q}}(t)-M_{p \bar{q}}(s)\right)\left(M_{\bar{p} q}(t)-M_{\bar{p} q}(s)\right) .
\end{aligned}
$$

Elementary, though tedious, consideration of all possible cases reveals that indeed any quadruple $\left(i_{p}, j_{q}, i_{\bar{p}}, j_{\bar{q}}\right)$ can be reduced by the above three transformations to one of the quadruples mentioned in the statement of Proposition 3.4.

It is worth noting that cases (i) and (ii) are not independent. First, they both apply if $1 \leq i_{p}<i_{\bar{p}} \leq n_{1}<j_{\bar{q}}<j_{q} \leq n$; the expressions prescribed by (3.7) and (3.8) are distinct, but yield the same result:

$$
\begin{aligned}
2 M_{p \bar{q}}(s) M_{\bar{p} q}(t) & -\frac{2 s}{t-s}\left(M_{p \bar{q}}(t) M_{\bar{p} q}(s)-M_{p \bar{q}}(s) M_{\bar{p} q}(t)\right) \\
& =2 M_{p \bar{q}}(t) M_{\bar{p} q}(s)-\frac{2 t}{t-s}\left(M_{p \bar{q}}(t) M_{\bar{p} q}(s)-M_{p \bar{q}}(s) M_{\bar{p} q}(t)\right) .
\end{aligned}
$$

Moreover, the expression for $n_{1}<i_{p}<i_{\bar{p}}<j_{\bar{q}}<j_{q} \leq n$ in case (ii) can be obtained from the expressions for $1 \leq i_{p}<i_{\bar{p}}<j_{\bar{q}}<j_{q} \leq n_{1}$ in case (i) by reversing the direction of the cut. However, we think that the above presentation, though redundant, better emphasizes the underlying symmetries of the expressions obtained.

The proof of relations (3.7)-(3.10) is similar to the proof of Theorem 3.3 in [GSV3] and is done by induction on the number of internal vertices in $N$. The key ingredient of the proof is the following straightforward analog of Lemma 3.5 from [GSV3].

Consider an arbitrary boundary vertex $b_{i}$ (without loss of generality we may assume that $b_{i}$ lies on the outer circle of the annulus) and suppose that the neighbor of $b_{i}$ is a black vertex $u$. Denote by $u_{+}$the unique vertex in $G$ such that $\left(u, u_{+}\right) \in E$, and by $u_{-}$ the neighbor of $u$ distinct from $u_{+}$and $b_{i}$. Create a new network $\widehat{N}$ by deleting $b_{i}$ and the edge $e_{0}=\left(b_{i}, u\right)$ from $G$, splitting $u$ into one new source $b_{i_{u}}$ and one new sink $b_{j_{u}}$ placed on the outer circle (so that either $i-1<i_{u}<j_{u}<i+1$ or $i-1<j_{u}<i_{u}<i+1$ ) and replacing the edges $e_{+}=\left(u, u_{+}\right)$and $e_{-}=\left(u_{-}, u\right)$ by new edges $\hat{e}_{+}=\left(b_{i_{u}}, u_{+}\right)$ and $\hat{e}_{-}=\left(u_{-}, b_{j_{u}}\right)$ (see Figure 3.3). We may assume without loss of generality that the cut $\rho$ in $N$ does not intersect the edge $e_{0}$, and hence $\rho$ remains a valid cut in $\widehat{N}$. 


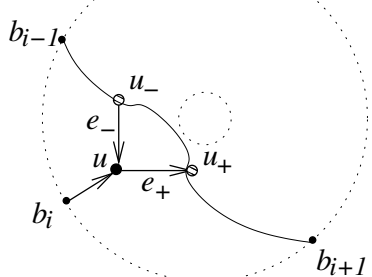

$N$

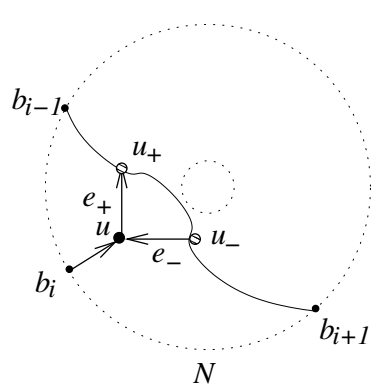

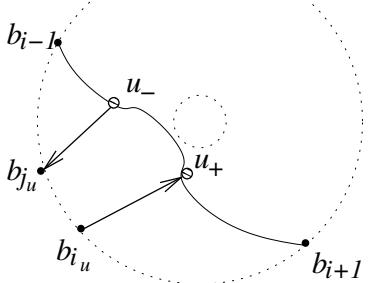

$\widehat{N}$

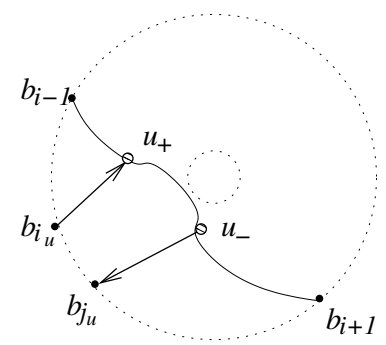

$\widehat{N}$

Fig. 3.3. Splitting a black vertex: cases $i-1<i_{u}<j_{u}<i+1$ (top) and $i-1<j_{u}<i_{u}<i+1$ (bottom).

Lemma 3.5. Boundary measurements in the networks $N$ and $\widehat{N}$ are related by

$$
\begin{aligned}
M\left(i_{p}, j\right) & =\frac{w_{e_{0}} w_{e_{+}} \widehat{M}\left(i_{u}, j\right)}{1+w_{e_{-}} w_{e_{+}} \widehat{M}\left(i_{u}, j_{u}\right)}, \\
M\left(i_{\bar{p}}, j\right) & =\widehat{M}\left(i_{\bar{p}}, j\right) \pm \frac{w_{e_{-}} w_{e_{+}} \widehat{M}\left(i_{\bar{p}}, j_{u}\right) \widehat{M}\left(i_{u}, j\right)}{1+w_{e_{-}} w_{e_{+}} \widehat{M}\left(i_{u}, j_{u}\right)}, \quad \bar{p} \neq p ;
\end{aligned}
$$

in the second formula above, the + sign corresponds to the cases

$$
i_{p}-1 \prec j_{u} \prec i_{u} \prec i_{p}+1 \preceq j \prec i_{\bar{p}} \quad \text { or } \quad i_{\bar{p}} \prec j \preceq i_{p}-1 \prec i_{u} \prec j_{u} \prec i_{p}+1,
$$

and the - sign corresponds to the cases

$$
i_{p}-1 \prec i_{u} \prec j_{u} \prec i_{p}+1 \preceq j \prec i_{\bar{p}} \quad \text { or } \quad i_{\bar{p}} \prec j \preceq i_{p}-1 \prec j_{u} \prec i_{u} \prec i_{p}+1,
$$

where $\prec$ is the cyclic order $\bmod n$.

We leave the details of the proof to the interested reader.

Denote by $\{\cdot, \cdot\}_{N}^{2}$ the member of the 2-parameter family (3.5), (3.6) corresponding to the case $\alpha=\beta=1$. Moreover, define $\sigma_{\times}\left(i, j, i^{\prime}, j^{\prime}\right)=\operatorname{sign}\left(i^{\prime}-i\right)+\operatorname{sign}\left(j^{\prime}-j\right)$; clearly, $\sigma_{\times}\left(i, j, i^{\prime}, j^{\prime}\right)$ is closely related to $s_{\times}\left(i, j, i^{\prime}, j^{\prime}\right)$ defined and studied in [GSV3]. The bracket induced by $\{\cdot, \cdot\}_{N}^{2}$ on the image of the boundary measurement map is completely described by the following statement. 
Proposition 3.6. (i) Let $1 \leq \max \left\{i_{p}, i_{\bar{p}}\right\}<j_{\bar{q}}<j_{q} \leq n$. Then

$$
\left\{M_{p q}(t), M_{\bar{p} \bar{q}}(s)\right\}_{N}^{2}=\sigma_{\times}\left(i_{p}, j_{q}, i_{\bar{p}}, j_{\bar{q}}\right) M_{p q}(t) M_{\bar{p} \bar{q}}(s)-2 \Gamma_{p q}^{\bar{p} \bar{q}}(t, s),
$$

where

$$
\Gamma_{p q}^{\bar{p} \bar{q}}(t, s)= \begin{cases}0, & j_{q} \leq n_{1}, \\ -s M_{p q}(t) M_{\bar{p} \bar{q}}^{\prime}(s), & j_{\bar{q}} \leq n_{1}<j_{q}, \\ t M_{p q}^{\prime}(t) M_{\bar{p} \bar{q}}(s)-s M_{p q}(t) M_{\bar{p} \bar{q}}^{\prime}(s), & \max \left\{i_{p}, i_{\bar{p}}\right\} \leq n_{1}<j_{\bar{q}}, \\ t M_{p q}^{\prime}(t) M_{\bar{p} \bar{q}}(s), & i_{p} \leq n_{1}<i_{\bar{p}},\end{cases}
$$

and $M_{p q}^{\prime}, M_{\bar{p} \bar{q}}^{\prime}$ are the derivatives of $M_{p q}$ and $M_{\bar{p} \bar{q}}$.

(ii) Let $1 \leq i_{p}<j_{\bar{q}}<i_{\bar{p}}<j_{q} \leq n$ and either $j_{\bar{q}} \leq n_{1}<i_{\bar{p}}$ or $j_{q} \leq n_{1}$. Then

$$
\left\{M_{p q}(t), M_{\bar{p} \bar{q}}(s)\right\}_{N}^{2}=0 .
$$

(iii) Let $1 \leq i_{p}<j_{q}<i_{\bar{p}}<j_{\bar{q}} \leq n$ and either $j_{q} \leq n_{1}<i_{\bar{p}}$ or $j_{\bar{q}} \leq n_{1}$. Then

$$
\left\{M_{p q}(t), M_{\bar{p} \bar{q}}(s)\right\}_{N}^{2}=0 .
$$

Proof. The proof is similar to the proof of Proposition 3.4. We leave the details to the interested reader.

Remark 3.7. It is worth mentioning that the bracket induced on $k \times m$ matrices via perfect planar networks in a disk, which was studied in [GSV3], can be considered as a particular case of (3.7)-(3.10) (for $\alpha=-\beta=1$ ) or (3.11)-(3.13) (for $\alpha=\beta=1$ ). To see this it suffices to consider only networks without edges that intersect the cut $\rho$, and to cut the annulus along $\rho$ in order to get a disk.

\subsection{Realization theorem}

To conclude the proof of Theorem 3.3 we need the following statement. We say that $F \in \mathrm{Rat}_{k, m}$ is represented by a network $N$ if $F$ belongs to the image of $M_{N}$.

Theorem 3.8. For any $F \in \operatorname{Rat}_{k, m}$ there exists a network $N \in \operatorname{Net}_{I_{1}, J_{1}, I_{2}, J_{2}}$ such that $F$ is represented by $N$.

Proof. We first prove the following simple observation concerning perfect planar networks in a disk.

Lemma 3.9. Let $n=4, I=\{1,2\}, J=\{3,4\}$. There exists a network $N_{\mathrm{id}} \in \operatorname{Net}_{I, J}$ such that the $2 \times 2$ identity matrix is represented by $N_{\mathrm{id}}$.

Proof. The proof is furnished by the network depicted in Fig. 3.4. The corresponding boundary measurement matrix is given by

$$
\left(\begin{array}{cc}
w_{1} w_{8}\left(w_{3} w_{11}\left(w_{2}+w_{6} w_{9} w_{10}\right)+w_{6} w_{7} w_{9}\right) & w_{1} w_{3} w_{4}\left(w_{2}+w_{6} w_{9} w_{10}\right) \\
w_{1} w_{6} w_{8}\left(w_{7}+w_{3} w_{10} w_{11}\right) & w_{3} w_{4} w_{5} w_{6} w_{10}
\end{array}\right)
$$

which yields the identity matrix for $w_{5}=w_{10}=-1$ and $w_{i}=1$ for $i \neq 5,10$. 


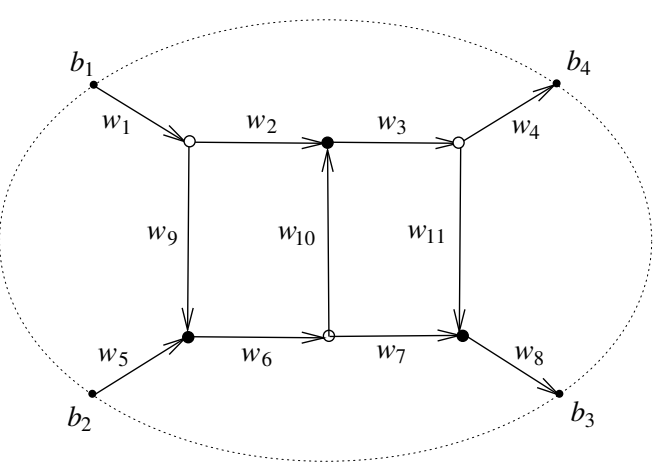

Fig. 3.4. The network $N_{\text {id }}$.

Effectively, Lemma 3.9 says that the planarity restriction can be omitted in the proof of Theorem 3.8. Indeed, if $F \in \mathrm{Rat}_{k, m}$ is represented by a non-planar perfect network in an annulus, one can turn it into a planar perfect network in an annulus by replacing each intersection by a copy of $N_{\text {id }}$.

In what follows we make use of the concatenation of planar networks in an annulus. Similarly to the case of networks in a disk, the most important particular case of concatenation arises when the sources and the sinks are separated, that is, all sources lie on the outer circle, and all sinks lie on the inner circle. We can concatenate two networks of this kind, one with $k$ sources and $m$ sinks and another with $m$ sources and $l$ sinks, by gluing the sinks of the former to the sources of the latter. More exactly, we glue together the inner circle of the former network and the outer circle of the latter in such a way that the corresponding base points of the cuts are identified, and the $i$ th sink of the former network is identified with the $(m+1-i)$ th source of the latter. The erasure of the common boundary and the identification of edges are performed exactly as in the case of a disk.

Let us start by representing any rational function $F \in$ Rat $_{1,1}$ by a network with the only source on the outer circle and the only sink on the inner circle.

Lemma 3.10. Any rational function $F \in \mathrm{Rat}_{1,1}$ can be represented by a network $N \in$

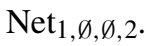

Proof. First, if networks $N_{1}, N_{2} \in \mathrm{Net}_{1, \emptyset, \emptyset, 2}$ represent functions $F_{1}$ and $F_{2}$ respectively, their concatenation $N_{1} \circ N_{2} \in \operatorname{Net}_{1, \emptyset, \emptyset, 2}$ represents $F_{1} F_{2}$.

Second, define the direct sum $N_{1} \oplus N_{2} \in \operatorname{Net}_{[1,2], \emptyset, \emptyset,[3,4]}$ as shown in Fig. 3.5 (left). The shadowed annuli contain networks $N_{1}$ and $N_{2}$. The intersections of the dashed parts of additional edges with the edges of $N_{1}$ and $N_{2}$ are resolved with the help of $N_{\text {id }}$ (not shown). Note that this direct sum operation is not commutative. Clearly, $N_{1} \oplus N_{2}$ represents the $2 \times 2$ matrix $\left(\begin{array}{cc}0 & F_{1} \\ F_{2} & 0\end{array}\right)$. The direct sum of networks is used to represent the sum $F_{1}+F_{2}$ as shown in Fig. 3.5 (right).

Third, if $N \in \operatorname{Net}_{1, \emptyset, \emptyset, 2}$ represents $F$, the network shown in Fig. 3.6 represents the function $F /(1+F)$, and, with a simple adjustment of weights, can also be used to represent $-F /(1+F)$. Taking the direct sum with the trivial network representing 1 , we get a representation for $1 /(1+F)$. 

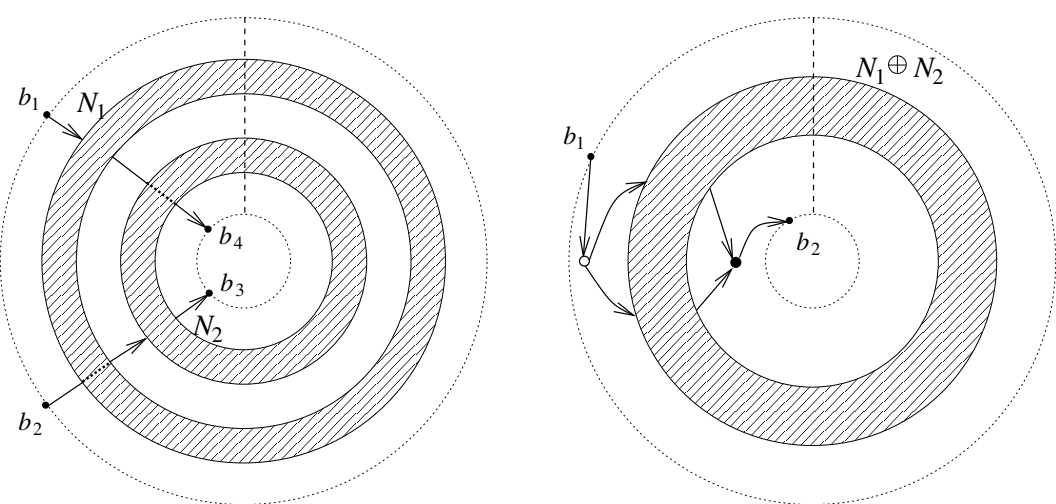

Fig. 3.5. The direct sum of two networks (left) and a network representing the sum of two functions (right).

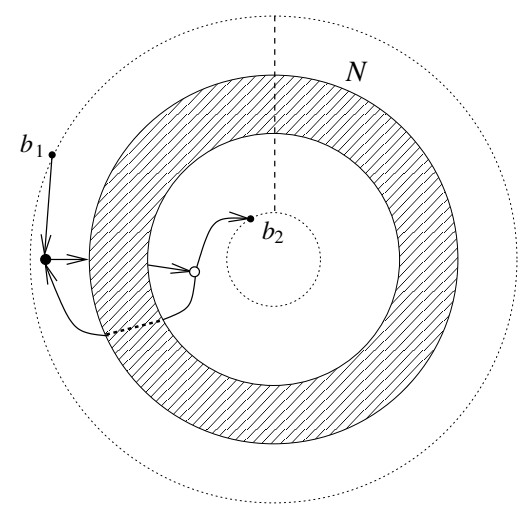

Fig. 3.6. Representing $F /(1+F)$.

Finally, the functions $a \lambda^{k}$ for any integer $k$ can be represented by networks in $\operatorname{Net}_{1, \emptyset, \emptyset, 2}$. The cases $k=2$ and $k=-2$ are shown in Fig. 3.7. Other values of $k$ are obtained in the same way.

We now have all the ingredients for the proof of the lemma. Any rational function $F$ can be represented as $F(\lambda)=\sum_{i=0}^{r} a_{i} \lambda^{d+i} / Q(\lambda)$, where $d$ is an integer and $Q$ is a polynomial satisfying $Q(0)=1$. Therefore, it suffices to represent each of the summands, and to use the direct sum construction. Each summand, in its turn, is represented by the concatenation of a network representing $a_{i} \lambda^{d+i}$ with a network representing $1 / Q=$ $1 /(1+(Q-1))$. The latter network is obtained as explained above from a network representing $Q-1=\sum_{j=1}^{p} b_{j} \lambda^{j}$ via the direct sum construction.

To get an analog of Lemma 3.10 for networks with the only source and the only sink on the outer circle, one has to use Lemma 3.9 once again. 

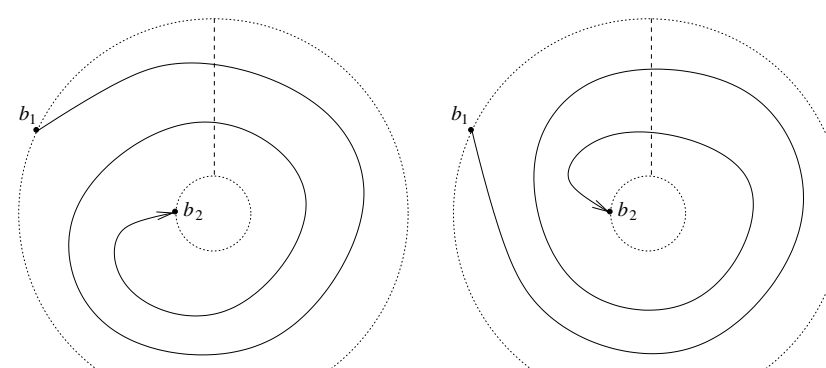

Fig. 3.7. Representing $a \lambda^{k}$ for $k=2$ (left) and $k=-2$ (right).

Lemma 3.11. Any rational function $F \in \mathrm{Rat}_{1,1}$ can be represented by a network $N \in$

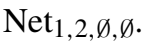

Proof. Such a representation is obtained from the one constructed in the proof of Lemma 3.10 by replacing the edge incident to the sink with a new edge sharing the same tail. The arising intersections, if any, are resolved with the help of $N_{\text {id }}$. For example, representation of $a(1+b \lambda)^{-1}$ obtained this way is shown in Fig. 3.8 (right). It makes use of the network $N_{\text {id }}$ described in Lemma 3.9; the latter is shown in thin lines inside a dashed circle. Note that the network on the left, which represents $a(1+b \lambda)^{-1}$ in $\operatorname{Net}_{1, \emptyset, \emptyset, 2}$, is not the one built in the proof of Lemma 3.10.
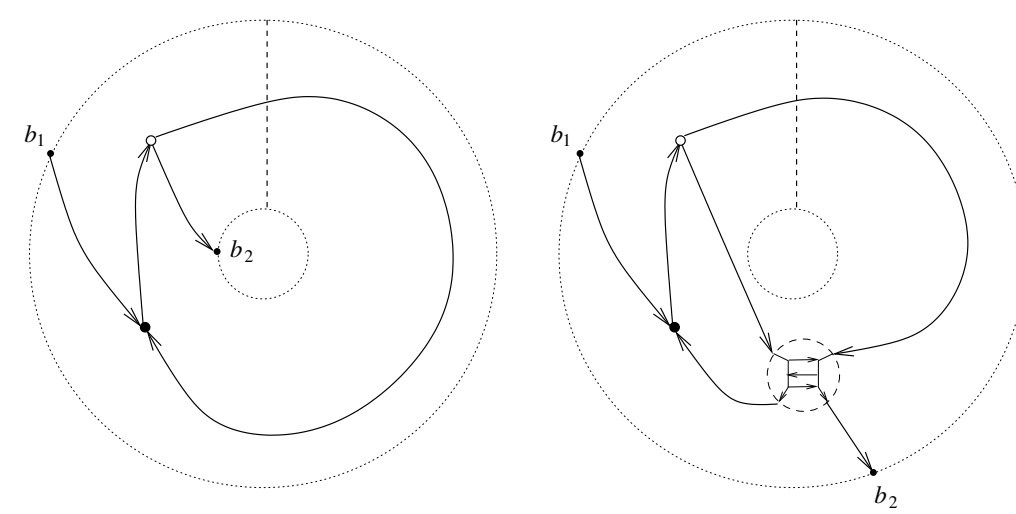

Fig. 3.8. Representing $a(1+b \lambda)^{-1}$ by a network in $\operatorname{Net}_{1, \emptyset, \emptyset, 2}$ (left) and $\mathrm{Net}_{1,2, \emptyset, \emptyset}$ (right).

Representation of rational functions by networks in $\mathrm{Net}_{2,1, \emptyset, \emptyset}$ and $\mathrm{Net}_{\emptyset, 1,2, \varnothing}$ is obtained in a similar way. In the latter case one has to replace also the edge incident to the source with a new one sharing the head.

The next step is to prove Theorem 3.8 in the case when all sources lie on the outer circle and all sinks lie on the inner circle. 
Lemma 3.12. For any rational matrix $F \in \operatorname{Rat}_{k, m}$ there exists a network $N \in$ $\mathrm{Net}_{[1, k], \emptyset, \emptyset,[k+1, k+m]}$ such that $F$ is represented by $N$.

Proof. First of all, we represent $F$ as $F=A \widetilde{F} B$, where $A=\left\{a_{i j}\right\}$ is the $k \times k m$ constant matrix given by

$$
a_{i j}= \begin{cases}1 & \text { if }(k-i) m<j \leq(k-i+1) m \\ 0 & \text { otherwise }\end{cases}
$$

$\widetilde{F}$ is the $k m \times k m$ diagonal matrix

$$
\widetilde{F}=\operatorname{diag}\left\{F_{k m}, F_{k, m-1}, \ldots, F_{k 1}, F_{k-1, m}, \ldots, F_{11}\right\},
$$

and $B$ is the $k m \times m$ constant matrix

$$
B=\left(\begin{array}{c}
W_{0} \\
\vdots \\
W_{0}
\end{array}\right)
$$

with $W_{0}=\left(\delta_{i, m+1-j}\right)_{i, j=1}^{m}$. Similarly to the case of networks in a disk, the concatenation of networks representing matrices $F_{1}$ and $F_{2}$ produces a network representing $F_{1} W_{0} F_{2}$. Therefore, in order to get $F$ as above, we have to represent the matrices $A, W_{0} \widetilde{F}$ and $W_{0} B$.

The first representation is achieved trivially as the disjoint union of networks representing the $1 \times m$ matrix $\left(\begin{array}{llll}1 & 1 & \ldots\end{array}\right)$; in fact, since $A$ is constant, it can be represented by a network in a disk. The second representation is obtained as the direct sum of networks representing each $F_{i j}$. Finally, the third representation can also be achieved by a network in a disk, via a repeated use of the network $N_{\text {id }}$.

Observe that in order to represent a $k \times m$ matrix we have to use intermediate matrices of a larger size.

To complete the proof of Theorem 3.8 we rely on Lemma 3.12 and use the same idea of replacing edges incident to boundary vertices as in the proof of Lemma 3.11.

So, Theorem 3.8 has been proved, and hence the proof of Theorem 3.3 is completed.

As we already mentioned in the proof, the 2-parameter family of Poisson brackets on Rat $_{k, m}$ induced by $(\alpha-\beta)\{\cdot, \cdot\}_{N}^{1}+(\alpha+\beta)\{\cdot, \cdot\}_{N}^{2}$, where $\{\cdot, \cdot\}_{n}^{1}$ and $\{\cdot, \cdot\}_{n}^{2}$ are described in Propositions 3.4 and 3.6, respectively, is denoted $\{\cdot, \cdot\}_{I_{1}, J_{1}, I_{2}, J_{2}}$.

\subsection{Recovering the trigonometric R-matrix bracket on $\mathrm{Rat}_{k, k}$}

As an application of the results obtained in Section 3.2, consider the set $N_{[1, k], \emptyset, \emptyset,[k+1,2 k]}$ of perfect networks with $k$ sources on the outer circle and $k$ sinks on the inner circle. Clearly, in this case the boundary measurement map takes $\mathcal{E}_{N}$ to Rat ${ }_{k, k}$. Just as we did in the proof of Theorem 3.8, we can replace $M_{N}$ with $A_{N}=M_{N} W_{0}$ and observe that the concatenation $N$ of networks $N_{1}, N_{2} \in N_{[1, k], \emptyset, \emptyset,[k+1,2 k]}$ leads to $A_{N}=A_{N_{1}} A_{N_{2}}$. We would like to take a closer look at the bracket $\{\cdot, \cdot\}_{N}^{1}$ in this case. 
First, recall (see, e.g., [FT]) that the space Rat $_{k, k}$ can be equipped with an R-matrix (Sklyanin) Poisson bracket

$$
\{A(t), A(s)\}=[R(t, s), A(s) \otimes A(t)],
$$

where the left-hand side should be understood as

$$
\{A(t), A(s)\}_{p \bar{p}}^{q \bar{q}}=\left\{a_{p q}(t), a_{\bar{p} \bar{q}}(s)\right\}
$$

and the R-matrix $R(t, s)$ is an operator acting in $\mathbb{R}^{k} \otimes \mathbb{R}^{k}$ that depends on parameters $t, s$ and solves the classical Yang-Baxter equation. Of interest to us is a bracket (3.14) that corresponds to the so-called trigonometric R-matrix [BD]

$$
R(t, s)=\frac{t+s}{s-t} \sum_{i=1}^{k} E_{i i} \otimes E_{i i}+\frac{2}{s-t} \sum_{1 \leq l<m \leq k}\left(t E_{l m} \otimes E_{m l}+s E_{m l} \otimes E_{l m}\right) .
$$

The bracket (3.14), (3.15) can be rewritten in terms of the matrix entries of $A(t)$ as follows (we only list non-zero brackets): for $p<\bar{p}$ and $q<\bar{q}$,

$$
\begin{aligned}
& \left\{a_{p q}(t), a_{\bar{p} \bar{q}}(s)\right\}=2 \frac{t a_{p \bar{q}}(s) a_{\bar{p} q}(t)-s a_{p \bar{q}}(t) a_{\bar{p} q}(s)}{t-s} . \\
& \left\{a_{p \bar{q}}(t), a_{\bar{p} q}(s)\right\}=2 t \frac{a_{p q}(s) a_{\bar{p} \bar{q}}(t)-a_{p q}(t) a_{\bar{p} \bar{q}}(s)}{t-s} . \\
& \left\{a_{p q}(t), a_{p \bar{q}}(s)\right\}=\frac{(t+s) a_{p \bar{q}}(s) a_{p q}(t)-2 s a_{p \bar{q}}(t) a_{p q}(s)}{t-s} . \\
& \left\{a_{p q}(t), a_{\bar{p} q}(s)\right\}=\frac{2 t a_{p q}(s) a_{\bar{p} q}(t)-(t+s) a_{p q}(t) a_{\bar{p} q}(s)}{t-s} .
\end{aligned}
$$

It is now straightforward to check that for $N \in N_{[1, k], \emptyset, \emptyset,[k+1,2 k]}$, the Poisson algebra satisfied by the entries of $A_{N}$ coincides with that of the Sklyanin bracket (3.14), (3.15). More exactly, relations (3.16) and (3.18) are equivalent to (3.7) with $\Phi_{p q}^{\bar{p} \bar{q}}(t, s)$ calculated according to the third case, while (3.17) and (3.19) are equivalent to (3.8) with $\Psi_{p q}^{\bar{p}} \bar{q}(t, s)$ calculated according to the first case. Finally, the brackets that vanish identically, correspond exactly to the situations listed in the second case in (3.9) and in the third case in (3.10).

To summarize, we have obtained the following statement.

Theorem 3.13. For any $N \in N_{[1, k], \emptyset, \emptyset,[k+1,2 k]}$ and any choice of parameters $\alpha_{i j}, \beta_{i j}$ in (3.2), (3.3) such that $\alpha=1$ and $\beta=-1$ in (3.4), the map $A_{N}:(\mathbb{R} \backslash 0)^{|E|} \rightarrow$ Rat $_{k, k}$ is Poisson with respect to the Sklyanin bracket (3.14) associated with the R-matrix (3.15).

Remark 3.14. Equations (3.16)-(3.19) can also be used to define a Poisson bracket in the "rectangular" case of Rat $k_{1}, k_{2}$. In this case, a concise description (3.14) of the bracket should be modified as follows:

$$
\{A(t), A(s)\}=R_{k_{1}}(t, s)(A(t) \otimes A(s))-(A(t) \otimes A(s)) R_{k_{2}}(t, s),
$$

where $R_{k_{i}}(t, s)$ denotes the R-matrix (3.15) acting in $\mathbb{R}^{k_{i}} \otimes \mathbb{R}^{k_{i}}$. 


\section{Poisson properties of the Grassmannian boundary measurement map}

\subsection{Grassmannian boundary measurement map and path reversal}

Let $N \in \operatorname{Net}_{I_{1}, J_{1}, I_{2}, J_{2}}$ be a perfect planar network in an annulus. Similarly to the case of a disk, we are going to provide a Grassmannian interpretation of the boundary measurement map defined by $N$. To this end, we extend the boundary measurement matrix $M_{N}$ to a $k \times n$ matrix $\bar{X}_{N}$ as follows:

(i) the $k \times k$ submatrix of $\bar{X}_{N}$ formed by $k$ columns indexed by $I=I_{1} \cup I_{2}$ is the identity matrix $\mathbf{1}_{k}$

(ii) for $p \in[1, k]$ and $j=j_{q} \in J$, the $(p, j)$-entry of $\bar{X}_{N}$ is $m_{p j}^{I}=(-1)^{s(p, j)} M_{p q}$, where $s(p, j)$ is the number of elements in $I$ lying strictly between $\min \left\{i_{p}, j\right\}$ and $\max \left\{i_{p}, j\right\}$ in the linear ordering; note that the sign is selected in such a way that the minor $\left(\bar{X}_{N}\right)_{[1, k]}^{I\left(i_{p} \rightarrow j\right)}$ coincides with $M_{p q}$, where $I\left(i_{p} \rightarrow j\right)=\left(I \backslash i_{p}\right) \cup j$.

We will view $\bar{X}_{N}$ as a matrix representative of an element $X_{N}$ in the space $L G_{k}(n)$ of rational functions $X: \mathbb{R} \rightarrow G_{k}(n)$. The latter space is called the space of Grassmannian loops, and the corresponding rational map $X_{N}: \mathcal{E}_{N} \rightarrow L G_{k}(n)$ is called the Grassmannian boundary measurement map.

Given a network $N$ and a simple path $P$ from a source $b_{i}$ to a sink $b_{j}$ in $N$, we define the reversal of $P$ as follows: for every edge $e \in P$, change its direction and replace its weight $w_{e}$ by $1 / w_{e}$; equivalently, the modified weight $\bar{w}_{e}$ is replaced by $1 / \bar{w}_{e}$. Clearly, after the reversal of $P$ all vertices preserve their color.

Denote by $N^{P}$ the network obtained from $N$ by the reversal of $P$, and by $R^{P}$ the corresponding path reversal map $\mathcal{E}_{N} \rightarrow \mathcal{E}_{N^{P}}$. Moreover, put $t^{P}=1$ if both endpoints of $P$ belong to the same boundary circle, and $t^{P}=-1$ otherwise. Define two maps from $L G_{k}(n)$ to itself: $S_{1}$ is the identity map, while $S_{-1}$ takes any $X(t) \in L G_{k}(n)$ to $X(-t)$. Our next goal is to prove that the path reversal map for paths not intersecting the cut commutes with the Grassmannian boundary measurement map up to $S_{t} P$.

Theorem 4.1. Let $P$ be a simple path from a source $b_{i}$ to a sink $b_{j}$ in $N$ such that $M(i, j)$ does not vanish identically and $P$ does not intersect the cut. Then

$$
S_{t} P \circ X_{N}=X_{N^{P}} \circ R^{P} .
$$

Proof. Let $I$ be the index set of the sources in $N$. The statement of the theorem is equivalent to the equality $S_{t} P\left(x_{K}\right) x_{I}^{P}=x_{K}^{P}$ for any subset $K \subset[1, n]$ of size $k$. Here and in what follows the superscript $P$ means that the corresponding value is related to the network $N^{P}$. The signs of the elements $m_{p j}^{I}$ are chosen in such a way that $x_{I}^{P}=M^{P}(j, i)$, so we have to prove

$$
S_{t} P\left(x_{K}\right) M^{P}(j, i)=x_{K}^{P} .
$$

The proof of (4.1) is by induction on the number of inner vertices in $N$.

Let us start with the case when $N$ does not have inner vertices. In this case it suffices to prove (4.1) with $K=I\left(i_{r} \rightarrow l\right)$ for all edges $e=\left(b_{i_{r}}, b_{l}\right)$. Assume first that the 
intersection index of each edge with the cut $\rho$ equals \pm 1 or 0 ; consequently, after a suitable isotopy, each edge either intersects $\rho$ exactly once, or does not intersect it at all. Let $e^{*}=\left(b_{i}, b_{j}\right)$ be the edge to be reversed, and hence ind $\left(e^{*}\right)=0$. If both $b_{i}$ and $b_{j}$ belong to the same boundary circle, then exactly the following two cases are prohibited:

$$
\begin{aligned}
& \min \left\{i_{r}, l\right\}<\min \{i, j\}<\max \left\{i_{r}, l\right\}<\max \{i, j\}, \\
& \min \{i, j\}<\min \left\{i_{r}, l\right\}<\max \{i, j\}<\max \left\{i_{r}, l\right\} .
\end{aligned}
$$

Consequently, reversing $e^{*}$ does not change $(-1)^{s(r, l)}$, which corresponds to the map $S_{1}$. If $b_{i}$ and $b_{j}$ belong to distinct boundary circles, then the above two cases are prohibited whenever $e$ does not intersect the cut. If $e$ intersects the cut then the above two cases are the only possibilities. Consequently, reversing $e^{*}$ does not change $(-1)^{s(r, l)}$ for the edges not intersecting the cut and reverses it for the edges intersecting the cut, which corresponds to the map $S_{-1}$.

It remains to lift the restriction on the intersection index of edges with $\rho$. If there exists an edge $e^{\prime}$ such that $\left|\operatorname{ind}\left(e^{\prime}\right)\right|>1$ then the endpoints of $e^{\prime}$ belong to distinct boundary circles, and for any other edge with the endpoints on distinct boundary circles, the intersection index with $\rho$ does not vanish. Consequently, only edges with the endpoints on the same boundary circle can be reversed, and the above reasoning applies, which leads to $S_{1}$.

Let now $N$ have inner vertices, and assume that the first inner vertex $v$ on $P$ is white. Denote by $e$ and $e^{\prime}$ the first two edges of $P$, and by $e^{\prime \prime}$ the third edge incident to $v$. In what follows we assume without loss of generality that the cut in $N$ does not intersect $e$. To find $M^{P}(j, i)$ consider the network $\widehat{N^{P}}$ that is related to $N^{P}$ exactly in the same way as the network $\widehat{N}$ defined immediately before Lemma 3.5 is related to $N$. Similarly to the first relation in Lemma 3.5, we find

$$
M^{P}(j, i)=\frac{w_{e}^{P} w_{e^{\prime}}^{P} \widehat{M^{P}}\left(j, j_{v}\right)}{1+w_{e^{\prime}}^{P} w_{e^{\prime \prime}}^{P} \widehat{M^{P}}\left(i_{v}, j_{v}\right)} .
$$

Taking into account that $w_{e}^{P}=1 / w_{e}, w_{e^{\prime}}^{P}=1 / w_{e^{\prime}}, w_{e^{\prime \prime}}^{P}=w_{e^{\prime \prime}}$, we finally get

$$
M^{P}(j, i)=\frac{\widehat{M^{P}}\left(j, j_{v}\right)}{w_{e} w_{e^{\prime}}+w_{e} w_{e^{\prime \prime}} \widehat{M^{P}}\left(i_{v}, j_{v}\right)} .
$$

To find $x_{K}^{P}$ we proceed as follows.

Lemma 4.2. Let the first inner vertex $v$ of $P$ be white. Then

$$
x_{K}^{P}= \begin{cases}\frac{w_{e^{\prime}}\left(\widehat{x^{P}}\right)_{K \cup i_{v}}+w_{e^{\prime \prime}}\left(\widehat{x^{P}}\right)_{K \cup j_{v}}}{w_{e^{\prime}}+w_{e^{\prime \prime}} \widehat{M^{P}}\left(i_{v}, j_{v}\right)} & \text { if } i \notin K, \\ \frac{\left(\widehat{x^{P}}\right)_{K\left(i \rightarrow j_{v}\right) \cup i_{v}}}{w_{e} w_{e^{\prime}}+w_{e} w_{e^{\prime \prime}} \widehat{M^{P}}\left(i_{v}, j_{v}\right)} & \text { if } i \in K .\end{cases}
$$

Proof. The proof utilizes explicit formulas (similar to those provided by Lemma 3.5) that relate boundary measurements in the networks $N^{P}$ and $\widehat{N^{P}}$. What is important, the \pm 
sign in the second formula in Lemma 3.5 and the sign $(-1)^{s(p, j)}$ defined at the beginning of this section interplay in such a way that any submatrix of $\bar{X}_{N^{P}}$ is the sum of the corresponding submatrix of $\bar{X}_{\widehat{N^{P}}}$ and a submatrix of the rank 1 matrix that is equal to the tensor product of the $i$ th column of $\bar{X}_{N^{P}}$ and the $j_{v}$ th row of $\bar{X}_{N^{P}}$.

To find $x_{K}$, create a new network $\tilde{N}$ by deleting $b_{i}$ and the edge $e$ from $G$, splitting $v$ into two sources $b_{i_{v}^{\prime}}, b_{i_{v}^{\prime \prime}}$ (so that either $i-1<i_{v}^{\prime}<i_{v}^{\prime \prime}<i+1$ or $i-1<i_{v}^{\prime \prime}<i_{v}^{\prime}<i+1$ ) and replacing the edges $e^{\prime}=\left(v, v^{\prime}\right)$ and $e^{\prime \prime}=\left(v, v^{\prime \prime}\right)$ by $\left(b_{i_{v}^{\prime}}, v^{\prime}\right)$ and $\left(b_{i_{v}^{\prime \prime}}, v^{\prime \prime}\right)$, respectively.

Lemma 4.3. Let the first inner vertex $v$ of $P$ be white. Then

$$
x_{K}= \begin{cases}w_{e} w_{e^{\prime}} \tilde{x}_{K \cup i_{v}^{\prime \prime}}+w_{e} w_{e^{\prime \prime}} \tilde{x}_{K \cup i_{v}^{\prime}} & \text { if } i \notin K, \\ \tilde{x}_{K\left(i \rightarrow i_{v}^{\prime}\right) \cup i_{v}^{\prime \prime}} & \text { if } i \in K .\end{cases}
$$

Proof. The proof is a straightforward computation.

By (4.2) and Lemmas 4.2 and 4.3, relation (4.1) boils down to

$$
S_{t} P\left(w_{e^{\prime}} \tilde{x}_{K \cup i_{v}^{\prime \prime}}+w_{e^{\prime \prime}} \tilde{x}_{K \cup i_{v}^{\prime}}\right) \widehat{M^{P}}\left(j, j_{v}\right)=w_{e^{\prime}}\left(\widehat{x^{P}}\right)_{K \cup i_{v}}+w_{e^{\prime \prime}}\left(\widehat{x^{P}}\right)_{K \cup j_{v}}
$$

for $i \notin K$, and

$$
S_{t} P\left(\widetilde{x}_{\left.K\left(i \rightarrow i_{v}^{\prime}\right) \cup i_{v}^{\prime \prime}\right)} \widehat{M^{P}}\left(j, j_{v}\right)=\widehat{\left(x^{P}\right.}\right)_{K\left(i \rightarrow j_{v}\right) \cup i_{v}}
$$

for $i \in K$. To prove these two equalities, we identify $b_{i_{v}^{\prime}}$ with $b_{j_{v}}$ and $b_{i_{v}^{\prime \prime}}$ with $b_{i_{v}}$. Under this identification we have $\widetilde{N^{P}}=\widehat{N^{P}}$, where $\widetilde{P}$ is the path from $b_{i_{v}^{\prime}}$ to $b_{j}$ in $\widetilde{N}$ induced by $P$. Observe that $\tilde{N}$ has fewer inner vertices than $N$, and that the index set of the sources in $\widetilde{N}$ is $I\left(i \rightarrow i_{v}^{\prime}\right) \cup i_{v}^{\prime \prime}$. Therefore, by the induction hypothesis,

$$
S_{t} \widetilde{P}\left(\widetilde{x}_{\widetilde{K}}\right) \widetilde{x}_{I\left(i \rightarrow i_{v}^{\prime}\right) \cup i_{v}^{\prime \prime}}=\widetilde{x}_{\widetilde{K}}^{\widetilde{P}}
$$

for any $\widetilde{K}$ of size $k+1$. Moreover, $\widetilde{x}_{I\left(i \rightarrow i_{v}^{\prime}\right) \cup i_{v}^{\prime \prime}}^{\widetilde{M}} \widetilde{M}^{\widetilde{P}}\left(j, i_{v}^{\prime}\right)=\widehat{M^{P}}\left(j, j_{v}\right)$ and $t^{P}=t^{\widetilde{P}}$. Therefore, using (4.3) for $\widetilde{K}=K \cup i_{v}^{\prime}=K \cup j_{v}, \widetilde{K}=K \cup i_{v}^{\prime \prime}=K \cup i_{v}$ and $\widetilde{K}=$ $K\left(i \rightarrow i_{v}^{\prime}\right) \cup i_{v}^{\prime \prime}=K\left(i \rightarrow j_{v}\right) \cup i_{v}$ we get both equalities above.

Assume now that the first inner vertex $v$ on $P$ is black. Denote by $e$ and $e^{\prime}$ the first two edges of $P$, and by $e^{\prime \prime}$ the third edge incident to $v$. To find $M^{P}(j, i)$, consider the network $\widetilde{N^{P}}$ similar to the one defined immediately before Lemma 4.3 , the difference being that the two new boundary vertices $j_{v}^{\prime}$ and $j_{v}^{\prime \prime}$ are sinks rather than sources. Clearly,

$$
M^{P}(j, i)=\frac{1}{w_{e} w_{e^{\prime}}}\left(\widetilde{M^{P}}\left(j, j_{v}^{\prime}\right)+w_{e^{\prime \prime}} w_{e^{\prime}} \widetilde{M^{P}}\left(j, j_{v}^{\prime \prime}\right)\right)
$$

To find $x_{K}^{P}$ we proceed as follows.

Lemma 4.4. Let the first inner vertex $v$ of $P$ be black. Then

$$
x_{K}^{P}= \begin{cases}\left(\widetilde{x^{P}}\right)_{K} & \text { if } i \notin K, \\ \frac{1}{w_{e} w_{e^{\prime}}}\left(\left(\widetilde{x^{P}}\right)_{K\left(i \rightarrow j_{v}^{\prime}\right)}+w_{e^{\prime \prime}} w_{e^{\prime}}\left(\widetilde{x^{P}}\right)_{K\left(i \rightarrow j_{v}^{\prime \prime}\right)}\right) & \text { if } i \in K .\end{cases}
$$

Proof. The proof is a straightforward computation. 
To find $x_{K}$ we consider the network $\widehat{N}$ defined immediately before Lemma 3.5.

Lemma 4.5. Let the first inner vertex $v$ of $P$ be black. Then

$$
x_{K}= \begin{cases}\frac{w_{e} w_{e^{\prime}} \widehat{x}_{K}}{1+w_{e^{\prime \prime}} w_{e^{\prime}} \widehat{M}\left(i_{v}, j_{v}\right)} & \text { if } i \notin K, \\ \frac{\widehat{x}_{K\left(i \rightarrow i_{v}\right)}+w_{e^{\prime \prime}} w_{e^{\prime}} \widehat{x}_{K\left(i \rightarrow j_{v}\right)}}{1+w_{e^{\prime \prime}} w_{e^{\prime}} \widehat{M}\left(i_{v}, j_{v}\right)} & \text { if } i \in K .\end{cases}
$$

Proof. The proof is similar to the proof of Lemma 4.2.

By (4.4) and Lemmas 4.4 and 4.5, relation (4.1) boils down to

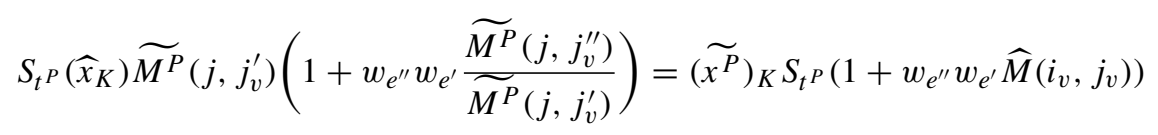

for $i \notin K$, and

$$
\begin{aligned}
S_{t} P\left(\widehat{x}_{K\left(i \rightarrow i_{v}\right)}\right. & +w_{e^{\prime \prime}} w_{\left.e^{\prime} \widehat{x}_{K\left(i \rightarrow j_{v}\right)}\right)} \widetilde{M^{P}}\left(j, j_{v}^{\prime}\right)\left(1+w_{e^{\prime \prime}} w_{e^{\prime}} \frac{\widetilde{M^{P}}\left(j, j_{v}^{\prime \prime}\right)}{\widetilde{M^{P}}\left(j, j_{v}^{\prime}\right)}\right) \\
& =\left(\left(\widetilde{x^{P}}\right)_{K\left(i \rightarrow j_{v}^{\prime}\right)}+w_{e^{\prime \prime}} w_{e^{\prime}}\left(\widetilde{x^{P}}\right)_{K\left(i \rightarrow j_{v}^{\prime \prime}\right)}\right) S_{t^{P}}\left(1+w_{e^{\prime \prime}} w_{e^{\prime}} \widehat{M}\left(i_{v}, j_{v}\right)\right)
\end{aligned}
$$

for $i \in K$. To prove these two equalities, we identify $b_{j_{v}^{\prime}}$ with $b_{i_{v}}$ and $b_{j_{v}^{\prime \prime}}$ with $b_{j_{v}}$. Under this identification we have $\widehat{N}^{\widehat{P}}=\widetilde{N^{P}}$, where $\widehat{P}$ is the path from $b_{i_{v}}$ to $b_{j}$ in $\widehat{N}$ induced by $P$. Observe that $\widehat{N}$ has fewer inner vertices than $N$, and that the index set of the sources in $\widehat{N}$ is $I\left(i \rightarrow i_{v}\right)$. Therefore, by the induction hypothesis,

$$
S_{t} P\left(\widehat{x}_{\widehat{K}}\right) \widehat{x}_{I\left(i \rightarrow i_{v}\right)}^{\widehat{P}}=\widehat{x}_{\widehat{K}}^{\widehat{P}}
$$

for any $\widehat{K}$ of size $k$. Taking into account that $\widehat{x}_{I\left(i \rightarrow j_{v}\right)}=\widehat{M}\left(i_{v}, j_{v}\right)$,

$$
\widehat{x}_{I\left(i \rightarrow i_{v}\right)}^{\widehat{P}}=\widehat{M}^{\widehat{P}}\left(j, j_{v}\right)=\widetilde{M^{P}}\left(j, j_{v}^{\prime \prime}\right), \quad \widehat{x}_{I\left(i \rightarrow j_{v}\right)}^{\widehat{P}}=\widehat{M}^{\widehat{P}}\left(j, i_{v}\right)=\widetilde{M^{P}}\left(j, j_{v}^{\prime}\right),
$$

and using (4.5) for $\widehat{K}=K, \widehat{K}=K\left(i \rightarrow i_{v}\right)=K\left(i \rightarrow j_{v}^{\prime}\right), \widehat{K}=K\left(i \rightarrow j_{v}\right)=$ $K\left(i \rightarrow j_{v}^{\prime \prime}\right)$ and $\widehat{K}=I\left(i \rightarrow j_{v}\right)$ we get both equalities above.

Remark 4.6. Theorem 4.1 is proved in $[\mathrm{P}]$ for networks in a disk. Observe that in this case $t^{P}$ vanishes identically, and hence $X_{N}$ and $R^{P}$ always commute.

\subsection{Induced Poisson structures on $L G_{k}(n)$}

Consider a subspace $L G_{k}^{I}(n) \subset L G_{k}(n)$ consisting of all $X \in L G_{k}(n)$ such that the Plücker coordinate $x_{I}$ does not vanish identically; clearly, $X_{N} \in L G_{k}^{I}(n)$. Therefore, we can identify $L G_{k}^{I}(n)$ with the space $\mathrm{Rat}_{k, m}$ equipped with the 2-parameter family of Poisson brackets $\{\cdot, \cdot\}_{I_{1}, J_{1}, I_{2}, J_{2}}$. 
The following result says that for any fixed $n_{1}=\left|I_{1}\right|+\left|J_{1}\right|$, the families of brackets $\{\cdot, \cdot\}_{I_{1}, J_{1}, I_{2}, J_{2}}$ on different subspaces $L G_{k}^{I}(n)$ can be glued together to form the unique 2-parameter family of Poisson brackets on $L G_{k}(n)$ that makes all maps $X_{N}$ Poisson.

Theorem 4.7. (i) For any fixed $n_{1}, 0 \leq n_{1} \leq n$, and any choice of parameters $\alpha$ and $\beta$ there exists a unique Poisson bracket $\mathcal{P}_{\alpha, \beta}^{n_{1}}$ on $L G_{k}(n)$ such that for any network $N$ with $n_{1}$ boundary vertices on the outer circle, $n-n_{1}$ boundary vertices on the inner circle, $k$ sources, $n-k$ sinks and weights defined by (3.1), the map $X_{N}:(\mathbb{R} \backslash 0)^{|E|} \rightarrow$ $L G_{k}(n)$ is Poisson provided the parameters $\alpha_{i j}$ and $\beta_{i j}$ defining the bracket $\{\cdot, \cdot\}_{N}$ on $(\mathbb{R} \backslash 0)^{|E|}$ satisfy relations (3.4).

(ii) For any $I \subset[1, n],|I|=k$, and any $n_{1}, 0 \leq n_{1} \leq n$, the restriction of $\mathcal{P}_{\alpha, \beta}^{n_{1}}$ to the subspace $L G_{k}^{I}(n)$ coincides with the bracket $\{\cdot, \cdot\}_{I_{1}, J_{1}, I_{2}, J_{2}}$ with $I_{1}=I \cap\left[1, n_{1}\right]$, $J_{1}=\left[1, n_{1}\right] \backslash I_{1}, I_{2}=I \backslash I_{1}, J_{2}=\left[n_{1}+1, n\right] \backslash I_{2}$.

Proof. This result is an analog of Theorem 4.3 proved in [GSV3], and one may attempt to prove it in a similar way. The main challenge in implementing such an approach is to check that the Poisson structures defined for two distinct subspaces $L G_{k}^{I}(n)$ and $L G_{k}^{I^{\prime}}(n)$ coincide on the intersection $L G_{k}^{I}(n) \cap L G_{k}^{I^{\prime}}(n)$. For the case of networks in an annulus, the direct check becomes too cumbersome. We bypass this difficulty in the following way.

Assume first that $\left|I \cap I^{\prime}\right|=k-1$ and take $i \in I \backslash I^{\prime}, j \in I^{\prime} \backslash I$. Denote by $\operatorname{Net}_{I_{1}, J_{1}, I_{2}, J_{2}}^{i j}$ the set of networks in $\mathrm{Net}_{I_{1}, J_{1}, I_{2}, J_{2}}$ satisfying the following two conditions: $M(i, j)$ does not vanish identically and there exists a path from $b_{i}$ to $b_{j}$ that does not intersect the cut. The set $\mathrm{Net}_{I_{1}^{\prime}, J_{1}^{\prime}, I_{2}^{\prime}, J_{2}^{\prime}}^{j i}$ is defined similarly, with the roles of $i$ and $j$ interchanged. Clearly, the path reversal introduced in Section 4.1 establishes a bijection between $\mathrm{Net}_{I_{1}, J_{1}, I_{2}, J_{2}}^{i j}$ and $\mathrm{Net}_{I_{1}^{\prime}, J_{1}^{\prime}, I_{2}^{\prime}, J_{2}^{\prime}}^{j i}$. Moreover, a suitable modification of Theorem 3.8 remains true for networks in $\mathrm{Net}_{I_{1}, J_{1}, I_{2}, J_{2}}^{i j}$ : these networks represent all rational matrix functions such that the corresponding component of the matrix does not vanish identically. To see that, we use the following construction. Let $v$ be the neighbor of $b_{i}$ in $N$ and $u$ be the neighbor of $b_{j}$ in $N$. Add two new white vertices $v^{\prime}$ and $v^{\prime \prime}$ and two new black vertices $u^{\prime}$ and $u^{\prime \prime}$. Replace the edge $\left(b_{i}, v\right)$ by the edges $\left(b_{i}, v^{\prime}\right)$ and $\left(v^{\prime}, v\right)$ so that the weight of the resulting path is equal to the weight of the replaced edge. In a similar way, replace $\left(u, b_{j}\right)$ by $\left(u, u^{\prime}\right)$ and $\left(u^{\prime}, b_{j}\right)$. Moreover, add edges $\left(v^{\prime}, v^{\prime \prime}\right)$ and $\left(u^{\prime \prime}, u^{\prime}\right)$ of weight 1 and two parallel edges $\left(v^{\prime \prime}, u^{\prime \prime}\right)$, one of weight 1 , and the other of weight -1 . Finally, resolve all the arising intersections with the help of the network $N_{\text {id }}$. Since the set of functions representable via networks in $\mathrm{Net}_{I_{1}, J_{1}, I_{2}, J_{2}}^{i j}$ is dense in the space of all rational matrix functions, the 2-parameter family $\{\cdot, \cdot \cdot\}_{I_{1}, J_{1}, I_{2}, J_{2}}$ is defined uniquely already by the fact that $M_{N}$ is Poisson for any $N \in \mathrm{Net}_{I_{1}, J_{1}, I_{2}, J_{2}}^{i j}$. Recall that the boundary measurement map $M_{N}$ factors through $\mathcal{F}_{N}$; clearly, the same holds for the Grassmannian boundary measurement map $X_{N}$. Moreover, the path reversal map $R^{P}$ commutes with the projection $y: \mathcal{E}_{N} \rightarrow \mathcal{F}_{N}$ and commutes with $X_{N}$ up to $S_{t} P$. Finally, Poisson brackets satisfying (3.7)-(3.10) and (3.11)-(3.13) commute with $S_{t} P$. Therefore, the Poisson structures $\{\cdot, \cdot\}_{I_{1}, J_{1}, I_{2}, J_{2}}$ and $\{\cdot, \cdot\}_{I_{1}^{\prime}, J_{1}^{\prime}, I_{2}^{\prime}, J_{2}^{\prime}}$ coincide on $L G_{k}^{I}(n) \cap L G_{k}^{I^{\prime}}(n)$. If $\left|I \cap I^{\prime}\right|=r<k-1$, 
we consider a sequence $\left(I=I^{(0)}, I^{(1)}, \ldots, I^{(k-r)}=I^{\prime}\right)$ such that $\left|I^{(t)} \cap I^{(t+1)}\right|=k-1$ for all $t=0, \ldots, k-r-1$ and apply to each pair $\left(I^{(t)}, I^{(t+1)}\right)$ the same reasoning as above.

Acknowledgments. We wish to express our gratitude to A. Postnikov who explained to us the details of his construction, and to V. Fock, S. Fomin and N. Reshetikhin for stimulating discussions. M. G. was supported in part by NSF Grants DMS \#0400484 and DMS \#0801204. M. S. was supported in part by NSF Grants DMS \#0401178 and PHY \#0555346. A. V. was supported in part by ISF Grant \#1032/08.

\section{References}

[BD] Belavin, A., Drinfeld, V.: Solutions of the classical Yang-Baxter equation for simple Lie algebras. Functional Anal. Appl. 16, 159-180 (1982) Zbl 0504.22016 MR 0674005

[BFZ] Berenstein, A., Fomin, S., Zelevinsky, A.: Parametrizations of canonical bases and totally positive matrices. Adv. Math. 122, 49-149 (1996) Zbl 0966.17011 MR 1405449

[B] Brenti, F.: Combinatorics and total positivity. J. Combin. Theory Ser. A 71, 175-218 (1995) Zbl 0851.05095 MR 1342446

[FT] Faddeev, L., Takhtajan, L.: Hamiltonian Methods in the Theory of Solitons. Springer, Berlin (2007) Zbl 1111.37001 MR 2348643

[Fa] Fallat, S.: Bidiagonal factorizations of totally nonnegative matrices. Amer. Math. Monthly 108, 697-712 (2001) Zbl 1032.15015 MR 1865657

[FG1] Faybusovich, L., Gekhtman, M. I.: Elementary Toda orbits and integrable lattices. J. Math. Phys. 41, 2905-2921 (2000) Zbl 1052.37051 MR 1755477

[FG2] Faybusovich, L., Gekhtman, M. I.: Poisson brackets on rational functions and multiHamiltonian structure for integrable lattices. Phys. Lett. A 272, 236-244 (2000) Zbl 1115.37336 MR 1774784

[FZ] Fomin, S., Zelevinsky, A.: Total positivity: tests and parametrizations. Math. Inteligencer 22, 23-33 (2000) Zbl 1052.15500 MR 1745560

[GSV1] Gekhtman, M., Shapiro, M., Vainshtein, A.: Cluster algebras and Poisson geometry. Moscow Math. J. 3, 899-934 (2003) Zbl 1057.53064 MR 2078567

[GSV2] Gekhtman, M., Shapiro, M., Vainshtein, A.: Cluster algebras and Weil-Petersson forms. Duke Math. J. 127, 291-311 (2005) Zbl 1079.53124 MR 2130414

[GSV3] Gekhtman, M., Shapiro, M., Vainshtein, A.: Poisson geometry of directed networks in a disk. Selecta Math. 15, 61-103 (2009) Zbl 1186.53095 MR 2511199

[GSV4] Gekhtman, M., Shapiro, M., Vainshtein, A.: Generalized Bäcklund-Darboux transformations for Coxeter-Toda flows from cluster algebra perspective. Acta Math. 206, 245-310 (2011) Zbl pre05963661

[GSV5] Gekhtman, M., Shapiro, M., Vainshtein, A.: Inverse problem for networks on a cylinder. In preparation

[GrSh] Grünbaum, B., Shephard, G.: Rotation and winding numbers for planar polygons and curves. Trans. Amer. Math. Soc. 322, 169-187 (1990) Zbl 0722.51012 MR 1024774

[KM] Karlin, S., McGregor, J.: Coincidence probabilities. Pacific J. Math. 9, 1141-1164 (1959) Zbl 0092.34503 MR 0114248

[LP] Lam, T., Pylyavskyy, P.: Total positivity in loop groups I: whirls and curls. arXiv:0812.0840 
[M] Moser, J.: Finitely many mass points on the line under the influence of the exponential potential - an integrable system. In: Dynamical Systems, Theory and Applications, Lecture Notes in Phys. 38, Springer, Berlin, 467-497 (1975) Zbl 0323.70012 MR 0455038

[P] Postnikov, A.: Total positivity, Grassmannians and networks. arXiv:math/0609764

[S] Scott, J.: Grassmannians and cluster algebras. Proc. London Math. Soc. 92, 345-380 (2006) Zbl 1088.22009 MR 2205721 